\title{
Der Computer als Denkzeug für hermeneutische Arbeit
}

\author{
Reinhard Keil, Paderborn
}

DOI: $10.25366 / 2020.89$

Zitation: Reinhard Keil, „Der Computer als Denkzeug für hermeneutische Arbeit”, in: Brückenschläge zwischen Musikwissenschaft und Informatik. Theoretische und praktische Aspekte der Kooperation, in Verbindung mit der Fachgruppe Digitale Musikwissenschaft hrsg. von Stefanie Acquavella-Rauch, Andreas Münzmay und Joachim Veit (= Musikwissenschaft: Aktuelle Perspektiven. Bericht über die Jahrestagung der Gesellschaft für Musikforschung 2019 in Paderborn und Detmold, Bd. 3), Detmold, Musikwissenschaftliches Seminar der Universität Paderborn und der Hochschule für Musik Detmold, 2020, S. 3-26, DOI: 10.25366/2020.89 


\title{
Der Computer als Denkzeug für hermeneutische Arbeit
}

\author{
REINHARD KEIL, PADERBORN
}

\section{Einleitung}

Bezüglich ihrer Techniken und Methoden scheint die Informatik kaum Berührungspunkte mit den Geisteswissenschaften zu besitzen. Steht im Ingenieurbereich die mathematische Modellierung von Informatiksystemen im Zentrum der wissenschaftlichen Fundierung, sind es in den Geisteswissenschaften Methoden und Verfahren mit einem starken hermeneutischen Charakter. Mit dem Entstehen der digitalen Geisteswissenschaften (Digital Humanities, DH) bricht die Welt der formalen Modellierung in einen Bereich ein, in dessen Methoden die Urteilskraft und die kritische Reflexion des Menschen die essenziellen Qualitätssicherungsmechanismen verkörpern.

Wurde zu Beginn noch die Frage der Inhaltsneutralität einzelner Werkzeuge oder Algorithmen diskutiert, ${ }^{1}$ steht mittlerweile die weitreichende Umgestaltung von Infrastrukturen auf der Tagesordnung, denn der Medienwechsel von analogen Einschreibtechnologien zu digitalen Medien berührt sämtliche Bereiche der Editorik, die durch vielschichtige Wechselwirkungen zwischen Technik und ihrem Einsatzumfeld und teilweise gegensätzlichen Interessen der beteiligten Akteure und Institutionen gekennzeichnet sind. ${ }^{2}$ Der damit verbundene Wandel kann nur inter- bzw. transdisziplinär bewältigt werden und liefert damit auch einen wesentlichen Begründungszusammenhang für ein Fachgebiet digitale Geisteswissenschaften.

Jedoch muss man konstatieren, dass die beteiligten Disziplinen bislang kaum kohärente, theoretisch fundierte Grundlagen für die anwendungsbezogene, aber nicht anwendungsspezifische Gestaltung technischer Systeme und Infrastrukturen vorweisen können. Das ist aber erforderlich, um im transdisziplinären Diskurs die Anschlussfähigkeit von Begriffen, Methoden und Techniken zu den jeweils anderen Disziplinen sichern zu können.

Mit dem Einsatz von Technik sind vielfältige Erwartungen verbunden. Es zeigt sich aber, dass es nicht durchgängig möglich ist, in einem eindeutigen oder gar formal beschreibbaren Verfahren Intentionen der Techniknutzung auf Merkmale und Eigenschaften einer technischen Lösung abzubilden. Das liegt zum einen an der Natur der Entwicklungsprozesse, die nachfolgend

1 Siehe dazu die "Thesen: Digital Humanities 2020", die für die Diskussion während der 1. Mitgliederversammlung des Verbands Digital Humanities im deutschsprachigen Raum (DHd) in Passau erstellt wurden, <http://dig-hum. de/thesen-digital-humanities-2020> (12.06.2020).

2 Siehe Joachim Veit, „Es bleibt nichts, wie es war - Wechselwirkungen zwischen digitalen und ,analogen' Editionen", in: editio 24 (2010), S. 37-52; Patrick Sahle, Digitale Editionsformen, zum Umgang mit der Überlieferung unter den Bedingungen des Medienwandels, 3 Bände, Norderstedt 2013; Andreas Oberhoff, Revisionssicherheit von Forschungsdateninfrastrukturen am Beispiel digitaler Editionen, Dissertation Universität Paderborn 2020. 
kurz skizziert werden, zum anderen am Arbeitsgegenstand, in diesem Fall historisch-kritische Musikeditionen. Sowohl die Entwicklung von Software als auch die hermeneutisch geprägte Arbeit von Editoren können aufgrund der Offenheit und der Unsicherheit, die diesen Prozessen innewohnt, als Formen von Wissensarbeit charakterisiert werden. ${ }^{3}$

Da mit Projekten in den digitalen Geisteswissenschaften meist die Entwicklung, Erprobung und Validierung neuer technischer Systeme einhergeht, braucht es sowohl ein begriffliches als auch ein methodisches Instrumentarium für die Kooperation, das jeweils zu den anderen beteiligten Disziplinen anschlussfähig ist. Es soll helfen, das zu Beginn eines Projektes bestehende Antizipationsdefizit zu verringern. Aufgrund der technischen Möglichkeiten geht es dabei nicht mehr um die Gestaltung isoliert einzusetzender Werkzeuge, sondern zugleich um die Einbettung und den Ausbau in einer sich weiterentwickelnden digitalen Editionsinfrastruktur.

Aus der Sicht der Informatik wird ein Ansatz für eine hypothesengeleitete Technikgestaltung vorgestellt. Vor dem Hintergrund einer kontrastiven Begriffsbildung werden dabei grundlegende technische Potenziale identifiziert und Gestaltungsdimensionen vorgestellt, die es gestatten sollen, im transdisziplinären Diskurs Wechselwirkungen zwischen der Technik und ihrem Nutzungsumfeld transparent zu machen und Gestaltungskonflikte auszubalancieren.

\section{Gestaltung als Prozess}

Auch wenn Software als ausführbare Maschinensteuerung ein höchst materielles Produkt ist, liegen wesentliche Qualitäten in der Natur der Entwicklungs- und Nutzungsprozesse begründet. Das Wissen darüber kann nicht hinreichend oder vollständig auf Attribute oder Eigenschaften des jeweils zu gestaltenden Produkts zurückgeführt werden. Diese Produkt-ProzessKomplementarität hat Christiane Floyd ${ }^{4} 1981$ zum Anlass genommen, den Blickwinkel weg von den (formalen) Produkten und hin zu ihrer Einbettung in die informellen Prozesse der Verständnisbildung und der Kommunikation zu verschieben. Floyd zeigt auf, dass die zeitliche Strukturierung des Entwicklungsprozesses sich sinnfälliger Weise nicht an der Strukturierung des endgültigen Produkts orientieren sollte, wie das die Begriffe Top-Down- bzw. BottomUp-Entwicklung nahelegen. Vielmehr müsse Softwareentwicklung als komplexer kooperativer Lernprozess verstanden werden, in dem Zwischenergebnisse und Zwischenprodukte entstehen, wieder verworfen oder auch verfeinert und erweitert werden. Die dabei entstehenden

3 Im Weiteren spielen spezifische Prozesse der Wissensarbeit keine Rolle. Der Begriff wird hier verwendet, weil mit ihm ausgedrückt werden soll, dass die entsprechenden Arbeitsaufgaben zumindest in Teilen ergebnisoffen sind und die dafür erforderlichen Arbeitsprozesse nicht angemessen formal spezifizierbar sind; vgl. Reinhard Keil: „Unterstützung kontingenter Wissensarbeit. Ein Rahmenwerk für die Entwicklung digitaler Arbeitsumgebungen zur Unterstützung des Forschungsdiskurses in den Kulturwissenschaften", in: STUDIOLO. Kooperative Forschungsumgebungen in den eHumanities, hrsg. von Eva-Maria Seng, Reinhard Keil und Gudrun Oevel, Berlin und Boston 2018, S. 7-25.

4 Christiane Floyd, „Process-Oriented Approach to Software Development”, in: Systems Architecture, Proceedings of the 6th ACM European Regional Conference, Westbury House 1981, S. 285-294. 
Zwischenergebnisse sind für den Verständnisbildungsprozess wichtig, finden sich aber im endgültigen Produkt nicht oder nur implizit wieder. Entsprechend gehen dabei auch viele Begründungszusammenhänge wieder verloren. Das Erarbeiten der vielfältigen Annahmen und Begründungen bezeichnete Peter Naur ${ }^{5}$ als Theoriebildungsprozess und er kommt zu dem Schluss, dass das Wiederherstellen der Theorie lediglich aufgrund dokumentierter Entwurfsentscheidungen nicht möglich sei, denn sie sei letztlich nur in den Köpfen der Entwickler vorhanden. ${ }^{6}$

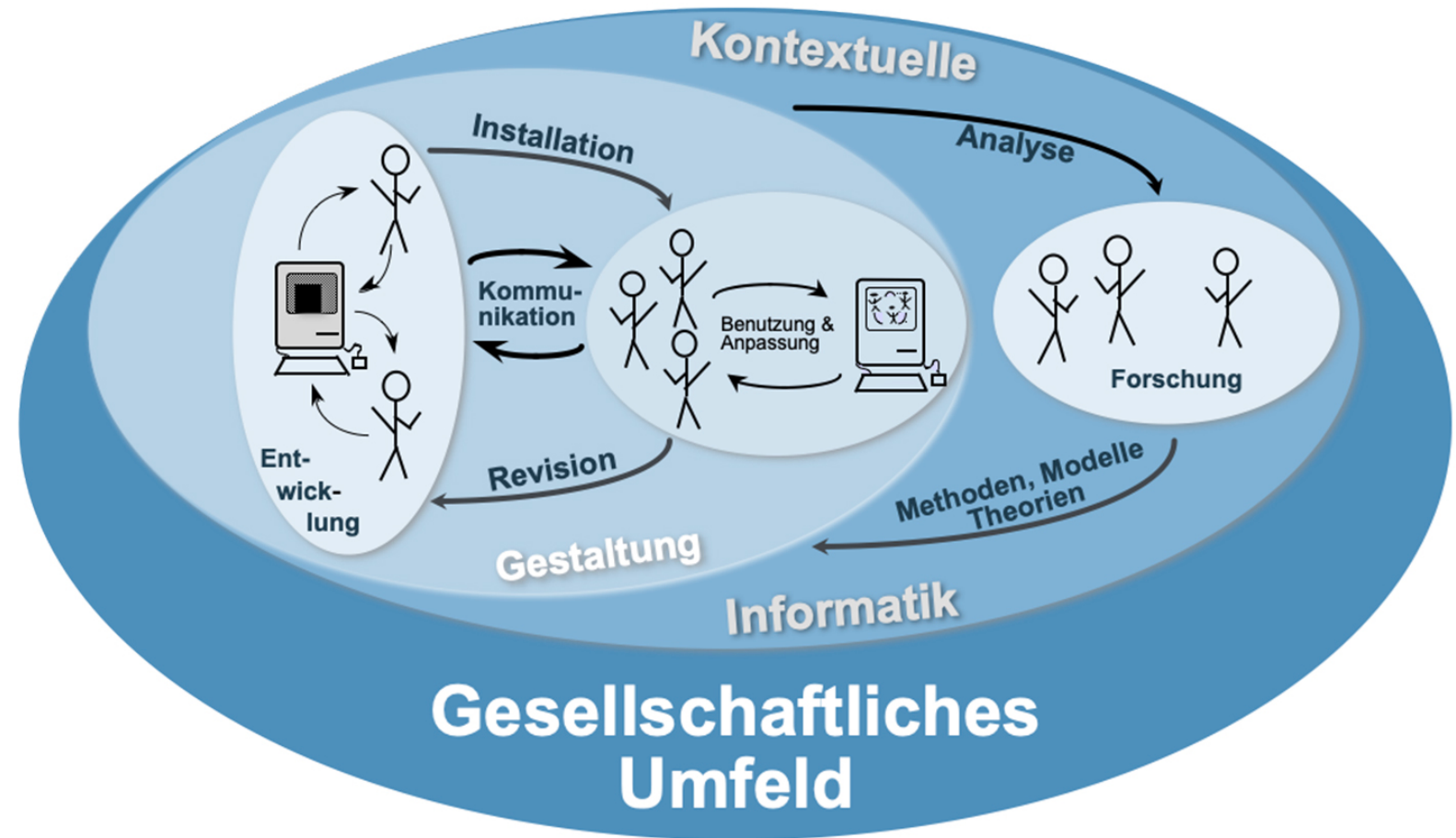

Abbildung 1: Lernzyklen bei der Systemgestaltung

Die Konsequenzen dieser Sicht werden schematisch vereinfacht in Abbildung 1 verdeutlicht: Der Verständnishorizont der jeweiligen Artefakte (Geräte, Dokumente, Methoden, Standards, etc.) ist in entsprechende Prozesse der Wissensarbeit eingebettet. Sie verkörpern in gewisser Weise immer nur Zwischenergebnisse im unaufhörlichen Wechselspiel von Analyse, Konstruktion, Bewertung und Weiterentwicklung. Die damit verbundenen Bedeutungen unterliegen dabei verschiedenen Perspektiven und Interessen und sind teilweise nur relativ zum jeweils

5 Peter Naur, „Programming as Theory Building”, in: Microprocessing and Microprogramming 15/5 (1985), S. 253261 <https://doi.org/10.1016/0165-6074(85)90032-8>; Nachdruck in der Sammlung von Aufsätzen Naurs: Computing: A Human Activity, ACM Press 1992.

6 Später entwickelte Techniken wie Prototyping (siehe Reinhard Budde et al. (Hrsg.), Approaches to Prototyping, Heidelberg 1983) und agile Projektmanagementtechniken (siehe Kent Beck, Extreme Programming Explained: Embrace Change, Boston 2000, sowie das agile Manifest unter <https://agilemanifesto.org>) tragen diesen Einsichten Rechnung. 
aktuellen oder einem vergleichbaren (Übertragbarkeit) Entwicklungs- und Nutzungskontext erschließbar.

Diese Sicht ist kompatibel mit konstruktivistischen Ansätzen und betont, dass ein Artefakt, gleich welcher Art, immer nur einen Anlass für menschliche Interpretationen verkörpert, die Bedeutung dabei aber nicht determiniert. ${ }^{7}$ Erst durch Normierung, standardisierten Gebrauch oder vorgegebene Interpretationsregeln lässt sich ein Ausschnitt an Bedeutungen gewissermaßen als Attribut oder Eigenschaft eines Artefakts oder Zeichens betrachten, die mit dem jeweiligen Artefakt weitergegeben werden kann. Letztlich ist es jedoch das interpretierende Bewusstsein des Menschen, das Bedeutung konstituiert.

Das stellt für Projekte in den digitalen Geisteswissenschaften im Allgemeinen und der $\mathrm{Mu}$ sikeditorik im Besonderen eine große Herausforderung dar. Zum einen ist der durch das technische System zu modellierende Gegenstandsbereich zu Projektbeginn noch nicht durchdrungen, zum anderen kommen mit den digitalen Medien neue Techniken und Verfahren ins Spiel, die bereits etablierte Normen und Praktiken durchbrechen sowie neue Möglichkeiten eröffnen.

Methodisch schaffen agile Entwicklungspraktiken eine gewisse Entlastung, indem durch erhöhten Kommunikationsaufwand und eine flexible Vorgehensweise in Ausbaustufen Fehlannahmen frühzeitig erkannt und der Aufwand für Korrekturen verringert werden sollen. Diese Kommunikationsprozesse leiden in dieser doppelten Lernsituation jedoch unter einem erheblichen Defizit: Im Extremfall wissen die Entwickler nicht, was die Forscher letztlich benötigen, und die Forscher können kaum antizipieren, welche technischen Möglichkeiten unter Alltagsbedingungen praktikabel sind und ihre Arbeit erleichtern. ${ }^{8}$ Das hat vielfältige Gründe, die vom Antizipieren der Wirkungen und Konsequenzen von Designentscheidungen über das Fehlen eines gemeinsamen Vokabulars bis hin zu Gestaltungskonflikten reichen, deren Auflösung in hohem Maße das Gewichten und Bewerten kontextabhängiger Faktoren erfordert.

Dies wird auch dadurch erschwert, dass sich die unterschiedlichen Forderungen an die Systemgestaltung nicht widerspruchsfrei umsetzen lassen. Es entstehen Designkonflikte, bei denen eine oder mehrere Anforderungen nur auf Kosten anderer, ebenso berechtigter Anforderungen umgesetzt werden können. Das Austarieren dieser Konflikte erfordert einen Ansatz, der über das klassische Repertoire der Softwaretechnik hinausgeht, denn die Konflikte können nur unter Bezug auf den Einsatzkontext angemessen aufgelöst werden. Dieser Einsatzkontext ist jedoch aufgrund der wechselseitigen Lernprozesse weder vollständig noch eindeutig modellierbar,

7 Dies gilt erst recht für die Modellierung von Softwaresystemen, die Christiane Floyd als Realitätskonstruktion charakterisiert (siehe Christiane Floyd, "A Comparative Evaluation of System Development Methods”, in: Information Systems Design Methodologies: Improving the Practice, hrsg. von T. William Olle, Henk G. Sol und Alex A. Verrijn-Stuart, Amsterdam 1986, S. 19-54; dies., "Outline of a Paradigm Change in Software Engineering", in: Computers and Democracy, A Scandinavian Challenge, hrsg. von Gro Bjerknes, Pelle Ehn, Morton Kyng, Avebury 1987, S. 185-202 und dies., "Softwareentwicklung als Realitätskonstruktion”, in: Software-Entwicklung: Konzepte, Erfahrungen, Perspektiven, Informatik-Fachberichte, hrsg. von Wolfram-M. Lippe, Berlin 1989, S. 1-20 sowie Christiane Floyd et al., Software Development and Reality Construction, Berlin 1992).

8 Aus diesem Dilemma heraus ist das Zentrum Musik - Edition - Medien (ZenMEM) gegründet worden, um Wissen, Werkzeuge und Methoden zugänglich zu machen und weiterzuentwickeln, vgl. <https://zenmem.de>. 
denn Lernprozesse finden kontinuierlich statt, neue Innovationen und Werkzeuge kommen hinzu, und die Vielfalt der Nutzungssituationen und die Individualität der Nutzer ist zu Beginn der technischen Entwicklung nur schwerlich vorhersehbar. Designentscheidungen müssen folglich vor dem Hintergrund unvollständigen und unsicheren Wissens getroffen werden. ${ }^{9}$

Die grundlegende Gestaltungshypothese lautet deshalb, dass die Qualität einer virtuellen Forschungsumgebung nicht in erster Linie durch eine Fülle von beziehungslos nebeneinanderstehenden Anforderungen bestimmt wird, sondern durch das angemessene Austarieren der vielfältigen dabei auftretenden Designkonflikte. In dieser Situation kann eine hypothesengeleitete Technikgestaltung helfen, die vielfältigen Kontextbezüge zu durchleuchten und Konfliktsituationen aufzudecken. Das ist nicht nur für die unmittelbare Systemgestaltung relevant, sondern kann auch helfen, informatisches Gestaltungswissen anschlussfähig zu gestalten. ${ }^{10}$

Ein wichtiger Punkt dabei ist, die Potenziale der Digitalisierung zunächst möglichst präzise aus technischer Sicht zu erfassen, um darüber Wirkungen und Forderungen zu identifizieren, die es gestatten, Hypothesen abzuleiten und damit auch die Auflösung von Designkonflikten zu unterstützen. ${ }^{11}$ Dabei ist es wichtig, zwischen technischen Potenzialen und Nutzungsformen zu unterscheiden, wenn es um neue mediale Qualitäten geht. Am Beispiel kooperativer Unterstützungsfunktionen, die häufig unter der Bezeichnung Web 2.0 zusammengefasst worden sind, verdeutlicht Sabrina Geißler, ${ }^{12}$ dass der Sekundärdiskurs - gestützt auf die traditionellen Instrumentarien der Informatik und der Medienwissenschaften - kaum in der Lage scheine, die neuen Qualitäten adäquat zu beschreiben. Insbesondere werde dabei nicht genügend deutlich zwischen der technischen Basis und der Dimension des Sozialen unterschieden. Das heißt, von der Technik werde allzu direkt auf soziale oder gesellschaftliche Folgen oder außertechnische Mehrwerte geschlossen.

\section{Kontrastive Begriffsbildung}

Um die Rolle des Computers in den digitalen Geisteswissenschaften präzisieren zu können, schlägt John Unsworth in einem Workshop des King's College im Jahr 2000 das Konzept der

9 Ausführlicher am Beispiel Software-Ergonomie in Reinhard Keil, „Hypothesengeleitete Technikgestaltung als Grundlage einer kontextuellen Informatik", in: Informationstechnik und ihre Organisationslücken. Soziale, politische und rechtliche Dimensionen aus der Sicht von Wissenschaft und Praxis, hrsg. von Andreas Breiter und Martin Wind, Berlin 2011, S. 165-184 und Reinhard Keil, Christian Schild, "Gestaltungskonflikte in der Softwareergonomie", in: Mensch \& Computer 2013 - Tagungsband. 13. fachübergreifende Konferenz für interaktive und kooperative Medien: Interaktive Vielfalt, hrsg. von Susanne Boll, Susanne Maaß und Rainer Malaka, München 2013, S. 67-77.

10 Siehe hierzu beispielhaft die Arbeit von Kai Holzweißig im Bereich kooperationsunterstützender Arbeitsumgebungen: Ein koaktiver Unterstützungsansatz für Prozesse sozialer Wirklichkeitskonstruktion in Produktentstehungsprozessen, Dissertation Universität Paderborn 2012.

11 Auch wenn eine strikte Trennung nicht möglich ist, müssen Nutzer und Entwickler bestrebt sein, die Konsequenzen von Gestaltungsentscheidungen in ihre jeweilige Handlungsdomäne zu übersetzen.

12 Sabrina Geißler, Mediale Destillation als innovative Qualität sozialer Software: ein informationstechnischer und medientheoretischer Ansatz zur Erschließung softwarebasierter Medien, Dissertation Universität Paderborn 2008. 
"Scholarly Primitives" vor. ${ }^{13}$ Damit bezeichnet er elementare, nicht weiter erklärungsbedürftige Funktionen, die in den verschiedenen geisteswissenschaftlichen Disziplinen gleichermaßen auftreten. In einem ersten Schritt schlägt er sieben solche Primitive vor: Discovering, Annotating, Comparing, Referring, Sampling, Illustrating und Representing. Sein Ziel ist es, ein handhabbares Rahmenwerk für die Verwaltung und koordinierte Entwicklung von digitalen Unterstützungswerkzeugen zu konzipieren und die Systematik entsprechend auch für die strukturierte Akquise von Forschungsprojekten einzusetzen.

Palmer et al. ${ }^{14}$ nutzen diesen Vorschlag, um den Diskurs in den Bibliothekswissenschaften über essentielle Informationsverarbeitungsaktivitäten zu strukturieren und Vergleiche zwischen verschiedenen Disziplinen zu ermöglichen. Blanke und Hedges ${ }^{15}$ gehen noch einen Schritt weiter und propagieren die Scholarly Primitives in einer modifizierten und erweiterten Form als Ausgangspunkt für den strukturierten Aufbau von digitalen Infrastrukturen, die den gesamten Lebenszyklus von Forschungsarbeiten in den digitalen Geisteswissenschaften umfassen und gewissermaßen eine digitale Produktionsstraße verkörpern sollen.

Zwar sind die Scholarly Primitives anwendungsbezogen aber nicht anwendungsspezifisch, doch sind sie für eine hypothesengeleitete Technikgestaltung nicht geeignet, weil eine klare Trennung zwischen geistigen Tätigkeiten, also der Wissensarbeit der Forscher (hermeneutische Arbeit), und technischen Operationen als Teil dieser Tätigkeiten nicht deutlich wird. Die unabhängige Beschreibung von Anforderungen und Systemleistungen ist für eine hypothesengeleitete Technikgestaltung aber unverzichtbar. Eine kontrastive Begriffsbildung, auch wenn sie nicht zum gängigen Kanon der Informatik gehört, könnte hier im interdisziplinären Diskurs verständnisbildend wirken und zugleich anschlussfähige Grundlagen für eine hypothesengeleitete Gestaltung liefern.

Beim Sprechen über digitale Technik und ihre Einsatzbereiche werden Begriffe aus dem einen Feld häufig im anderen Feld metaphorisch verwendet. Seit der Entwicklung des Computers sind metaphorische Konzepte aus dem Begriffsrepertoire der Informatik nicht mehr wegzudenken. Dabei steht die Analogie zwischen dem Computer und dem Gehirn bzw. algorithmischen Abläufen und kognitiven Prozessen im Vordergrund. Begriffe wie Semantik, Dialogsystem oder auch Künstliche Intelligenz verdeutlichen, dass entsprechende technische Konzepte in Anlehnung an Begriffe aus dem menschlichen Umfeld verstanden werden. Da diese Begriffe jedoch

13 John Unsworth, "Scholarly Primitives: What Methods Do Humanities Researchers Have in Common, and How Might Our Tools Reflect This", in: Humanities Computing: Formal Methods, Experimental Practice Symposium. King's College, London 2000, <http://www.people.virginia.edu/ jmu2m/Kings.5-00/primitives.html> (12.06.2020).

14 Carole L. Palmer, Lauren C. Teffeau u. Carrie M. Pirmann, Scholarly Information Practices in the Online Environment: Themes from the Literature and Implications for Library Service Development. Report commissioned by OCLC Research < https://www.oclc.org/content/dam/research/publications/library/2009/2009-02.pdf> (12.06.2020).

15 Tobias Blanke, Mark Hedges, „Humanities e-Science: From systematic investigations to institutional infrastructures", in: ESCIENCE '10: Proceedings of the 2010 IEEE Sixth International Conference on e-Science, S. 25-32 <https:// doi.org/10.1109/eScience.2010.34> (12.06.2020) und T. Blanke, M. Hedges, "Scholarly primitives: Building institutional infrastructure for humanities e-Science", in: Future Generation Computer Systems 29 (2013), S. 654-661. 
in den wissenschaftlichen Disziplinen teilweise sehr unterschiedlich verwendet werden, ist es nicht verwunderlich, dass beispielsweise in Bezug auf den Begriff der Interaktivität kaum zwei gleiche Definitionen in der Literatur zu finden sind. ${ }^{16}$ Für die Entwicklung eines hypothesengeleiteten Ansatzes, bei dem das technische Potenzial zur Unterstützung menschlicher Wissensarbeit im Vordergrund steht, ist das äußerst unbefriedigend.

Ohne klare begriffliche Unterscheidungen bzw. eine kontrastive Begriffsbildung zwischen den Leistungen des Computers und des Menschen lassen sich somit Potenziale nicht angemessen identifizieren. Dazu ein Beispiel. Der Begriff Kommunikation wird sowohl in der Sphäre der Technik als auch im Bereich zwischenmenschlicher Interaktion verwendet, bezeichnet aber nicht das Gleiche. Eine technische Kommunikationsfunktion gestattet es Nachrichten zwischen Sender und Empfänger auszutauschen. Dabei wird mit technischen Mitteln sichergestellt, dass die Nachrichten unverfälscht und vollständig übertragen werden, oder anders ausgedrückt, die technische Kommunikationsfunktion ist inhaltsneutral. Bei der zwischenmenschlichen Kommunikation wird die Bedeutung einer Nachricht u. a. auch durch den Kontext, die wechselseitigen Erwartungen der Kommunikationsteilnehmer und die Interpunktion vorangegangener Kommunikationshandlungen bestimmt. Für die technische Übertragung wird dagegen in der Regel ein gedächtnisloser Kanal genutzt, d. h. der Transportweg ist nach der Übertragung für den nächsten Kommunikationsvorgang (präziser: Übertragungsvorgang) wieder frei.

Natürlich lässt sich ein technisches System ausbauen, um z. B. eine Koordinierungsfunktion zu implementieren, mit deren Hilfe die beteiligten Akteure ihre Handlungen aufeinander abstimmen. Entscheidend für eine technische Koordinierungsfunktion ist, dass das technische System explizit spezielle Daten und Auswertemechanismen für die Koordinationsfunktion erhebt, speichert und auswertet. Nutzt ein Sender beispielsweise eine Kommunikationsfunktion um dem Empfänger mitzuteilen, dass sie sich zu einer bestimmten Uhrzeit an einem bestimmten Ort treffen wollen, haben sie keine technische Koordinierungsfunktion benutzt, denn die Tatsache, dass hier Handlungen koordiniert werden, basiert allein auf der Interpretation des Inhalts der Nachricht durch die beteiligten Akteure, nicht jedoch darauf, dass auf Seiten der Technik speziell dafür realisierte Funktionen eingesetzt würden. Dies wäre beispielsweise beim Einsatz eines digitalen Gruppenkalenders der Fall, der unter anderem entsprechende Benachrichtigungen wie z. B. Erinnerungen versendet und die gemeinsame Verwaltung und Änderungen der Daten ermöglicht. Diese zusätzlichen technischen Aktionen entlasten die Akteure auf unterschiedlichen Ebenen und eröffnen auch neue Handlungsmöglichkeiten, indem z. B. neuen Gruppenmitgliedern der Zugriff auf den gemeinsamen Kalender gewährt werden kann, statt mühsam aus den vielen vergangenen Nachrichten die für die Koordinierungsaktivitäten relevanten herauszufinden und erneut zu verschicken.

16 Siehe Felix Winkelnkemper, Responsive Positioning. A User Interface Technique Based on Structured Space, Dissertation Universität Paderborn 2017, S. 9-43. 
Bei der kontrastiven Begriffsbildung werden technische Potenziale über die physische Manipulation von Objekten definiert, nicht über spezifische Absichten und semantische Vorstellungen der Nutzer. Da Potenziale nicht anwendungsspezifisch sein sollen, werden nicht die individuellen kognitiven Fähigkeiten des Menschen betrachtet, sondern zunächst nur die grundlegende Architektur menschlicher Wahrnehmung, auf der alle höheren kognitiven Prozesse aufbauen. ${ }^{17}$

\section{Potenziale digitaler Medien}

Nachfolgend geht es um die Frage, welche Eigenschaften und Merkmale technischer Systeme das Potenzial haben, Mehrwerte in der Wissensarbeit zu erzielen. Entscheidend ist dabei, dass technische Potenziale sich im jeweiligen Nutzungskontext entfalten können, jedoch nicht notwendigerweise müssen. Technische Potenziale verkörpern demnach noch keinen Mehrwert; dieser entsteht erst durch die Einbettung in menschliches Handeln.

Aufgrund der in Abbildung 1 dargestellten Lernzyklen besteht bei der Gestaltung interaktiver Systeme grundsätzlich ein Antizipationsdefizit, das im Kontext hermeneutischer Arbeit noch verstärkt wird. Dieses Defizit soll durch Hypothesen nach Möglichkeit verringert werden, indem Potenziale identifiziert und die Wechselwirkungen mit unterschiedlichen Nutzungsformen untersucht werden. Das kann auch begriffliche Klarheit schaffen, denn für metaphorische Begriffe wie "interaktives System" oder auch "Interaktivität" lässt sich aufgrund der unzähligen unterschiedlichen Definitionen keine solche Analyse durchführen. ${ }^{18}$

Gemäß der Konzeption des Computers durch John von Neumann liegen Daten und die Programme zu ihrer Bearbeitung im Speicher des Rechners. Von außen sind diese nur mit großem Aufwand zugänglich. Zudem ist Wissen über die internen technischen Strukturen erforderlich. Wie Winkelnkemper ${ }^{19}$ herausarbeitet, entstehen allmählich zunehmend komplexere Schnittstellen, die es gestatten, die Elemente im Speicher mithilfe eigener kleiner Programme anzuzeigen und zu manipulieren. Interaktive Systeme stellen mit solchen Schnittstellen aus Programmen und (Daten-)Objekten den Nutzern eine eigene manipulierbare Welt zur Verfügung stellen, mit der sie Funktionen, Daten und Systemzustände sowohl einsehen als auch bearbeiten und verwalten können (siehe Abb. 2).

17 Siehe hierzu Keil, „Hypothesengeleitete Technikgestaltung” (wie Fn. 9) und ders., „Unterstützung kontingenter Wissensarbeit" (wie Fn. 3).

18 Im Bereich der Musik sei hier auf Jobst P. Fricke, „Musik: Analog-digital-analog. Digitalisierung und Begrifflichkeit als Norm in einer scheinbar analogen Welt", in: KlangArt-Kongreß 1995. Vorträge und Berichte, hrsg. von Bernd Enders und Niels Knolle (= Musik und Neue Technologie 1), Osnabrück 1995, S. 38-51 verwiesen, der Digitalisierung überwiegend als Diskretisierung versteht, u. a. um Parallelen zwischen der digitalen Welt der Computer und mentalen Prozessen aufzuzeigen.

19 Winkelnkemper, Responsive Positioning (wie Fn. 16). 


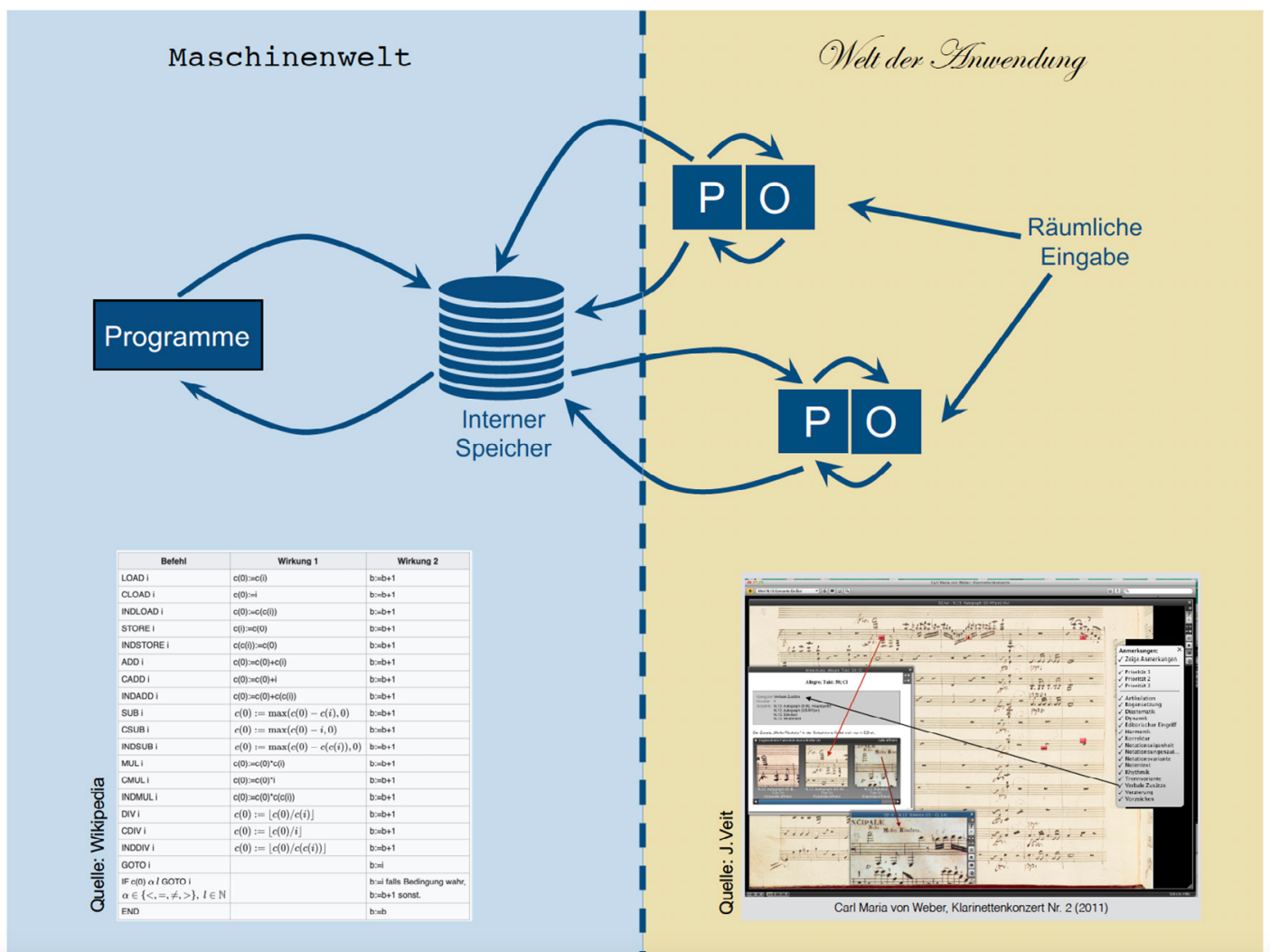

Abbildung 2: Eine Interaktive Schnittstelle stellt programmierte Funktionen und Objekte zur Verfügung (Quelle: F. Winkelnkemper, wie Fn. 16)

Die entscheidende Qualität interaktiver Systeme liegt also weniger im Sprachlichen (Dialogsystem) noch im Grafischen (bildhafte Darstellung), sondern im Objekthaften und damit zusammenhängend im Räumlichen. Interaktive Systeme bieten die Möglichkeit, Objekte jedweder Art (Funktionen, Zeichen, Bilder Dokumente, etc.) anzuzeigen, räumlich anzuordnen, zu platzieren und mit Hilfe direktmanipulativer Techniken zu bearbeiten. ${ }^{20}$

Darauf lassen sich zwei grundsätzliche Potenziale begründen:

Objekthaftigkeit:

Analoge Medientechniken sind Einschreibtechniken, d. h. mit der Erzeugung oder Aufzeichnung werden Zeichen fest mit dem Trägermaterial verbunden. Mit technischen Mitteln kann nur der Zeichenträger bearbeitet werden (z. B. Filmschnitt) nicht jedoch die jeweils semantisch bedeutsame Einheit. Auch digitale Medien sind grundsätzlich Einschreibtechnologien, bei denen Zahlenwerte, die die Objekte der Anwendung repräsentieren, in Speicherstellen geschrieben oder aus diesen ausgelesen werden. Durch die programmierte Schnittstelle sind Trägereigenschaften wie Adresse oder die Größe von

20 Ben Shneiderman prägte den Begriff „direct manipulation“ und charakterisierte sie durch folgende drei Merkmale: Objekte sind kontinuierlich sichtbar, Aktionen können inkrementell ausgeführt und zurückgenommen werden und geben kontinuierlich Rückmeldung; vgl. Shneiderman, "Direct manipulation: a step beyond programming languages", in: IEEE Computer, 16/8 (1983), S. 57-69, <https://doi.org/10.1109/MC.1983.1654471>. 
Speicherzellen weder bewusstseinspflichtig noch verkörpern sie Handlungseinheiten auf der Nutzungsebene. Wiederholte Einschreibprozesse im Speicher passieren so schnell, dass der Eindruck entsteht, tatsächlich ein Objekt am Bildschirm zu bewegen. Da das Zeichenarrangement variabel ist, können auch selektiv Zeichen eingefügt, gelöscht oder überschrieben und mit einem Objekt kombiniert oder in Teilobjekte aufgelöst werden.

Ko-aktives Schreiben:

Durch die Kopplung von Zeichen und Trägermaterial bei analogen Einschreibtechnologien haben mediale Objekte immer genau einen Ort, an dem sie physisch vorhanden sind und bearbeitet werden können. Zwar lassen sich mit entsprechendem Aufwand Vervielfältigungen erstellen, doch ist jede Bearbeitung wiederum an die lokale Kopie gebunden. Eine Synchronisation ist zwar möglich, aber mit einem hohen Aufwand verbunden, der durch einen Medienbruch hervorgerufen wird und nicht mit dem eigentlichen Arbeitsinhalt zusammenhängt. Mit digitalen Medien kann das Bewusstsein entsprechend entlastet werden, weil die Einschreib- und Übertragungsvorgänge miteinander kombiniert und so schnell ausgeführt werden, dass sie nicht mehr bewusstseinspflichtig sind. Es entsteht der Eindruck verteilter Persistenz, weil eine lokale Eingabe an einem Gerät zur Bearbeitung eines Objekts auf einem anderen Gerät an einem anderen Ort genutzt werden kann. Dieses technische Potenzial wird als ko-aktives Schreiben bezeichnet, um es von sozialen Formen der Kooperation oder Koordinierung abzugrenzen. Damit sind zum ersten Mal in unserer Kulturgeschichte Prozesse des gemeinsamen nebenläufigen Schreibens an verschiedenen Orten an ein- und demselben Dokument bzw. Objekt möglich. ${ }^{21}$

Das technische Potenzial wird über die Möglichkeiten des ko-aktiven Schreibens definiert und nicht über Begriffe wie Social Software oder Virtuelle Gemeinschaften. Letztere leiten sich in der Regel aus sozialen Nutzungsformen ab, in denen dieses technische Potenzial im Rahmen einer Kooperation einen Mehrwert entfalten kann. Doch worin der Mehrwert der Nutzung besteht, ist offen, denn ko-aktive Funktionen können auch im Rahmen einer strikten Arbeitsteilung mit hierarchischen Vorgaben einen Mehrwert erzielen, ohne dass sie im eigentlichen Sinn dadurch die Kooperation oder Kollaboration in einem Team unterstützen.

Im Kontext von Wissensarbeit ermöglichen Funktionen des ko-aktiven Schreibens den Aufbau geteilter Handlungs- und Wahrnehmungsräume in Form virtueller Wissensräume. ${ }^{22}$ Um die

21 Den mit ihnen verbundenen möglichen Mehrwert bezeichnet Geißler, Mediale Destillation (wie Fn. 12), als mediale Destillation.

22 Virtuelle Wissensräume müssten im Sinne einer kontrastiven Begriffsbildung korrekt als Wissensarbeitsräume bezeichnet werden, da sie im strikten Sinne kein Wissen verwalten, sondern lediglich einen (virtuellen) Ort für Dokumente und Objekte bereitstellen, die Anlass und Gegenstand von Wissensarbeit sind. Inwieweit dabei ein Objekt oder Dokument Wissen verkörpert, entscheidet sich im jeweiligen Nutzungskontext, nicht jedoch anhand der technischen Funktionen. Unterschiedliche Konzeptionen und Ausprägungen finden sich $u$. $a$. in Thorsten Hampel, Virtuelle Wissensräume - Ein Ansatz für die kooperative Wissensorganisation, Dissertation Universität Paderborn 2001; Jonas Schulte et al., „KoForum - Kooperative Forschungsumgebung für die organisationsüber- 
verschiedenen Ausprägungen systematisch aufbereiten zu können, ist es notwendig, sowohl einzelne Funktionen oder einzelne technische Potenziale isoliert zu betrachten. Einen Ansatz zur Systematisierung liefern die in Abbildung 3 dargestellten Gestaltungsdimensionen. ${ }^{23}$

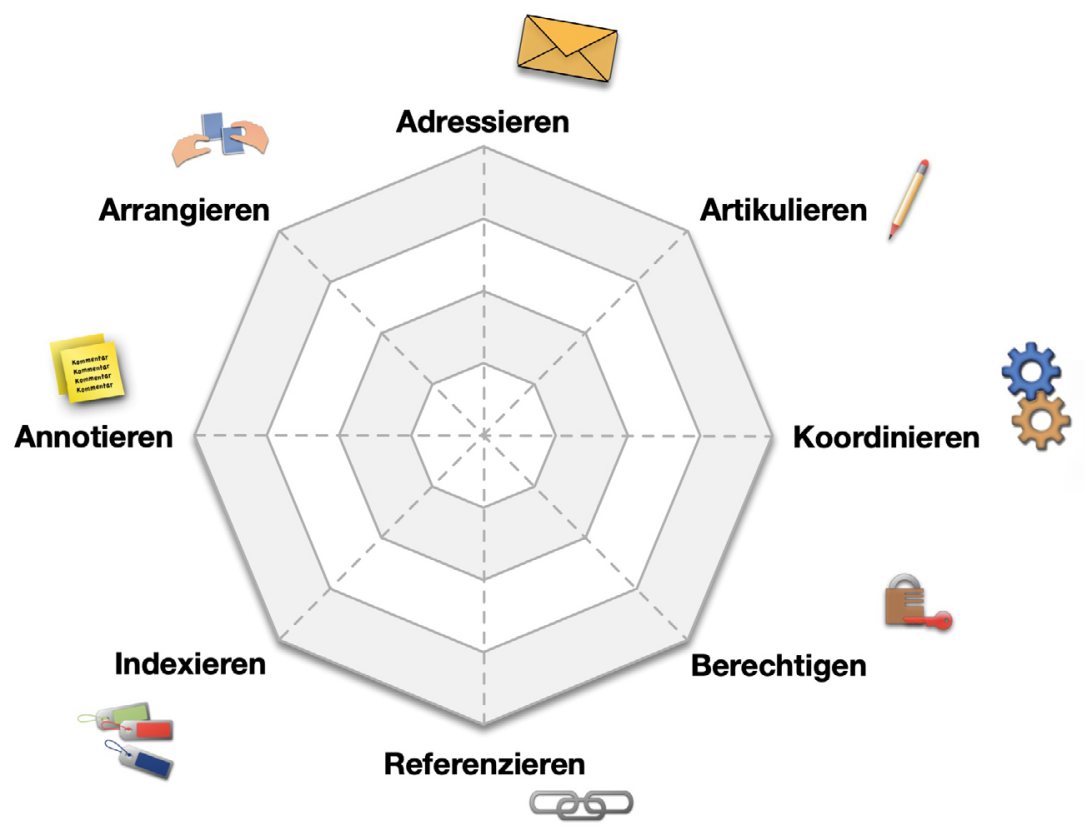

Abbildung 3: Gestaltungsdimensionen ko-aktiver Wissensräume

Eine Gestaltungsdimension ist gekennzeichnet durch einen Satz von Daten, die durch technische Funktionen erfasst, gespeichert und ausgewertet werden.

In vielen Anwendungen des WWW sind beispielsweise Verweise (Links) in einem Dokument integriert. Das führt dazu, dass das Potenzial des "nicht-sequentiellen Schreibens"24 nicht ausgenutzt wird, da für das Anbringen eines Verweises in einem Dokument Autorenrechte benötigt werden. Diese beziehen sich aber bei reinen Web-Seiten im HTML-Format jeweils auf das gesamte Dokument. Zwar ist der Verweis für die maschinelle Verarbeitung explizit syntaktisch gekennzeichnet, doch ist er als Objekt für einen anderen Autor oder Lesenden nicht zugäng-

greifende wissenschaftliche Laborarbeit", in: Wissensgemeinschaften: Digitale Medien - Öffnung und Offenheit in Forschung und Lehre, hrsg. von Thomas Köhler und Jörg Neumann, Münster 2011, S. 92-101; Reinhard Keil und Harald Selke, „Virtuelle Wissensräume - Von der Präsentation von Inhalten zu virtuellen Lernstätten”, in: 20 Jahre Lernen mit dem World Wide Web. Technik und Bildung im Dialog. Symposium. Heinz Nixdorf Institut, hrsg. von Reinhard Keil und Harald Selke, Paderborn 2015, S. 39-53, sowie Felix Winkelnkemper und Andreas Oberhoff, "WebArena - Räumliche Strukturen für die Lernorte der Zukunft", in: 20 Jahre Lernen mit dem World Wide Web, a. a. O., S. 103-121.

23 Diese Systematisierung hat sich analytisch bewährt. Unsere Arbeitshypothese lautet, dass sie auch konstruktiv bei der Systemgestaltung eingesetzt werden kann. Die hier skizzierte Ausprägung der Dimensionen basiert im Wesentlichen auf Arbeiten von Jonas Schulte.

24 So definierte Ted Nelson den von ihm geprägten Begriff Hypertext; vgl. Theodor Holm Nelson, Literary Machines, Sausalito 1982; Edition 87.1: Swarthmore PA 1987. 
lich. ${ }^{25}$ Handlungs- und Wahrnehmungsraum sind diesbezüglich getrennt. Erst wenn Verweise als eigenständige Objekte vom System verwaltet werden, ist es möglich, Berechtigungen nur für das Anbringen von Verweisen zu erteilen. Das Referenzieren stellt somit eine eigene Gestaltungsdimension dar, die es erst ermöglicht, unterschiedliche Formen des verteilten Schreibens zu realisieren.

Erst mit klaren begrifflichen Unterscheidungen ist es möglich, zwischen verschiedenen Systemarchitekturen oder Systemklassen und ihren jeweiligen Nutzungsformen zu differenzieren. Das hilft vor allem auch, das Gestaltungsdefizit zu reduzieren, wenn es dadurch gelingt, im Rahmen einer hypothesengeleiteten Gestaltung widersprüchliche Anforderungen aufzudecken, Gestaltungskonflikte auszutarieren oder auch neue Nutzungspotenziale aufzudecken.

Das Beispiel macht zugleich deutlich, dass die Gestaltungsdimensionen in praktischen Anwendungen miteinander verwoben sind, denn auch das Erteilen von Berechtigungen ist eine eigene Gestaltungsdimension. Um das Gestaltungspotenzial auszuschöpfen, müssen unterschiedliche Rechte festgelegt, verwaltet und überprüft werden können, d. h. es muss festgelegt sein, welche Objekte mit jeweils welcher Art von Berechtigung versehen werden können. ${ }^{26} \mathrm{Da}-$ bei lassen sich unterschiedliche Rechte und Strategien umsetzen. Neben klassischen Rechten wie Lesen oder Schreiben, kann z. B. auch ein Recht vorgesehen werden, das es den Nutzern gestattet, bei der Arbeit mit der Anwendung Berechtigungen an Dritte zu vergeben. Zusammen mit den Möglichkeiten Rechte-Hierarchien zu bilden oder auch Rechte temporär zu vergeben, lassen sich so unterschiedliche System-Architekturen entwickeln.

Die hier vorgestellten Gestaltungsdimensionen sind nicht abschließend gemeint. Sie sind im Kontext der Entwicklung kooperationsunterstützender Umgebungen entstanden und können je nach Erkenntnislage im Rahmen von Lernzyklen (Abbildung 2) variiert und erweitert werden. Aus dem Blickwinkel einer kontrastiven Begriffsbildung ist nicht entscheidend, was jeweils als Dimension abgegrenzt wird, sondern dass eine klare Differenzierung zwischen einer vorgeschlagenen Systemarchitektur und der Breite der damit unterstützten Nutzungsformen ermöglicht wird. Nur so können im Rahmen der Systemmodellierung die jeweiligen Vor- und Nachteile einer spezifischen Architektur erkannt und die Wechselwirkungen zwischen technischer Lösung und aktuellen Nutzungsanforderungen durchschaut werden.

$\mathrm{Zu}$ berücksichtigen ist noch, dass eine Gestaltungsdimension immer auch eine mehr oder weniger vertraute Nomenklatur für das Ansprechen der jeweiligen Objekte und Funktionen mit sich bringt, die erlernt werden muss. Zugleich müssen auch entsprechende Standards

25 Da die Objektwelten von Autoren und Lesern von Webseiten unterschiedlich sind, bezieht sich die Trennung von Handlungs- und Wahrnehmungsraum auf mögliche Anschlusshandlungen. So kann ein Autor auf der eigenen Seite zwar einen Verweis auf eine fremde Webseite anbringen, aber er kann auf der fremden Seite keine eigenen Verweise auf weitere Seiten oder einen Rückverweis auf die eigene Seite anbringen.

26 Ein differenziertes Beispiel zur Ausgestaltung virtueller Räume findet sich in Jonas Schulte et al., „Enhanced security management for flexible and dynamic cooperative environments", in: 2009 5th International Conference on Collaborative Computing, Networking, Applications and Worksharing: CollaborateCom 2009, Washington, D.C., November 11-14, 2009, IEEE, S. 1-10, <https://doi.org/10.4108/ICST.COLLABORATECOM2009.8377>. 
entwickelt und angepasst sowie Wissen über die neuen damit verbundenen Wirkungen erarbeitet werden. Wie der nächste Abschnitt zeigen soll, ist nicht nur dieser Zusatzaufwand zu bedenken, sondern auch die Tatsache, dass sich aus den verschiedenen Dimensionen in der Umsetzung eine Fülle neuer Gestaltungskonflikte ergeben.

\section{Gestaltung virtueller Forschungsumgebungen und -infrastrukturen}

Zeitdruck, begrenzte Ressourcen, pragmatische Lösungen oder interdisziplinäre Verständnisprobleme sind neben der Komplexität der zu bearbeitenden Aufgaben nur ein Teil der Probleme bei der Gestaltung von Arbeitsumgebungen für die Wissensarbeit. Die nachfolgend vorgestellte Sicht soll helfen, speziell im interdisziplinären Diskurs eine Brücke zwischen Ingenieur- und Geisteswissenschaften zu schlagen. Dabei steht die Anschlussfähigkeit auf Seiten der Technik im Vordergrund mit dem Ziel, die Gestaltungskompetenz zu erhöhen.

Mit Objekthaftigkeit und ko-aktivem Schreiben sind zwei grundlegende Potenziale digitaler Systeme vorgestellt worden. Interaktive Systeme, die dieses Potenzial auszuschöpfen versuchen, verkörpern einen physischen Handlungs- und Wahrnehmungsraum, der die Umgebung des Menschen um weitere mediale Objekte und Funktionen zu ihrer Bearbeitung und Verwaltung erweitert. Die grundlegende Gestaltungshypothese lautet: Je enger Handlungs- und Wahrnehmungsraum miteinander gekoppelt sind, je mehr also während der Nutzung Medienbrüche und Transformationsprobleme (z. B. die Umsetzung von einer Notation in eine andere) reduziert und dabei zugleich während der Nutzung die Objekte der Wahrnehmung mit möglichst geringem Aufwand zu Objekten des Handelns gemacht werden können, desto besser. Es wird damit kein konkretes Ziel spezifiziert, sondern eine Richtung für die Auflösung von Konflikten vorgegeben.

Dazu ein Beispiel. Beim Kollationieren, also dem Vergleich einer Urschrift mit einer Abschrift hinsichtlich Vollständigkeit und Korrektheit müssen die zu vergleichenden Objekte möglichst gleichzeitig im Wahrnehmungsfeld präsent sein (Schaffung eines Handlungs- und Wahrnehmungsraums). Da der Wahrnehmungsraum eines Menschen relativ eng begrenzt ist, ist zum Vergleichen das Hin- und Herspringen zwischen den Notenschriften erforderlich. Das ist sehr zeitaufwändig und auch fehleranfällig, weil z. B. Unterschiede bei längeren Texten oder Schriften übersehen werden können.

Um den Aufwand zu reduzieren und damit zugleich die Fehlerrate zu reduzieren, entwickelte Charlton Hinman Ende der 1940er Jahre ein Gerät zur optischen Unterstützung der Analyse (vgl. Abbildung 4).

Mithilfe einer trickreichen Anordnung von Spiegeln und optischen Linsen können zwei hinreichend gleiche Seiten ausgerichtet und übereinander projiziert werden. Durch zwei wechselnd blinkende Lichter fallen Unterschiede sofort ins Auge, weil nur die durch die wechselnde Beleuchtung deutlich werdenden Veränderungen einen Wahrnehmungsunterschied hervorru- 
fen. In Bezug auf die Wahrnehmung von rein optischen Unterschieden war damit eine enorme Zeitersparnis verbunden. ${ }^{27}$

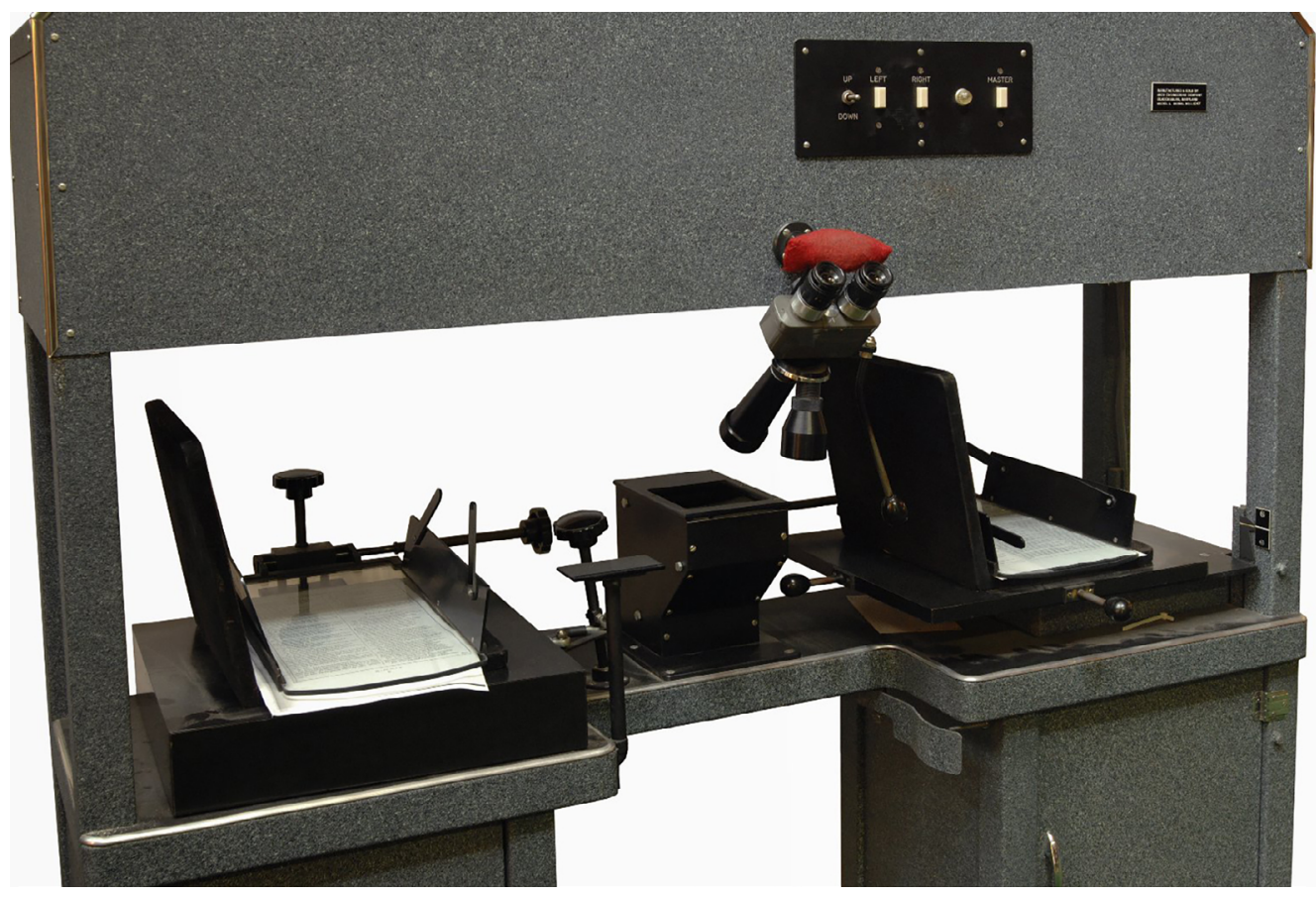

Abbildung 4: Der Hinman Collator der Folger Shakespeare Library ${ }^{28}$

Die Unterstützung bezieht sich bei diesem Gerät nur auf die Organisation des Wahrnehmungsfeldes. Es können keinerlei eigene Objekte kreiert werden; Annotationen müsse separat angelegt und verwaltet werden. Berücksichtigt man zusätzlich die Gestaltungsdimension Annotation, könnte man in interaktiven Umgebungen in einer ersten Stufe zumindest Anmerkungen vornehmen, die separat und ohne Schreibprozess auf die digitalisierte Quelle verarbeitet werden können. Die dazu erforderlichen Daten, wie z. B. der Inhalt der Annotation, die Referenz auf das Dokument und die Position im Dokument werden entsprechend im System separat verwaltet.

Eine weitere Gestaltungsdimension, die bislang in vielen Anwendungen noch stark vernachlässigt wird, ist das Arrangieren. Zwar lassen sich im Rahmen der Desktop-Metapher Schnittstellenobjekte arrangieren und auch anordnen, jedoch werden dabei keine Positionsdaten für die Objekte erhoben und verarbeitet. Ohne solche Positionsdaten ist es nicht möglich durch räumliches Arrangieren Attribute oder auch Werte von Objekten zu ändern. ${ }^{29}$

27 Für eine ausführliche Beschreibung sei auf auf Steven Escar Smith, „The Eternal Verities Verified: Charlton Hinman and the Roots of Mechanical Collation", in: Studies in Bibliography, hrsg. von Daivd L. van der Meulen, Band 53, Charlottesville 2000, S. 129-162 verwiesen.

28 Quelle: <https://upload.wikimedia.org/wikipedia/commons/0/0e/Hinman_collator.jpg>.

29 Das Potenzial einer räumlichen Zu- und Anordnung von Objekten bietet zwar erhebliches Potenzial für neue Formen interaktiven Arbeitens, doch wie Winkelnkemper, Responsive Positioning (wie Fn. 16) feststellt, lassen sich 
Die beiden Bilder in Abbildung 5 zeigen zwei Varianten eines partiell digitalisierten Arbeitsplatzes für Editoren, die im Rahmen von Forschungsarbeiten am ZenMEM konzipiert wurden und weiterentwickelt werden sollen.
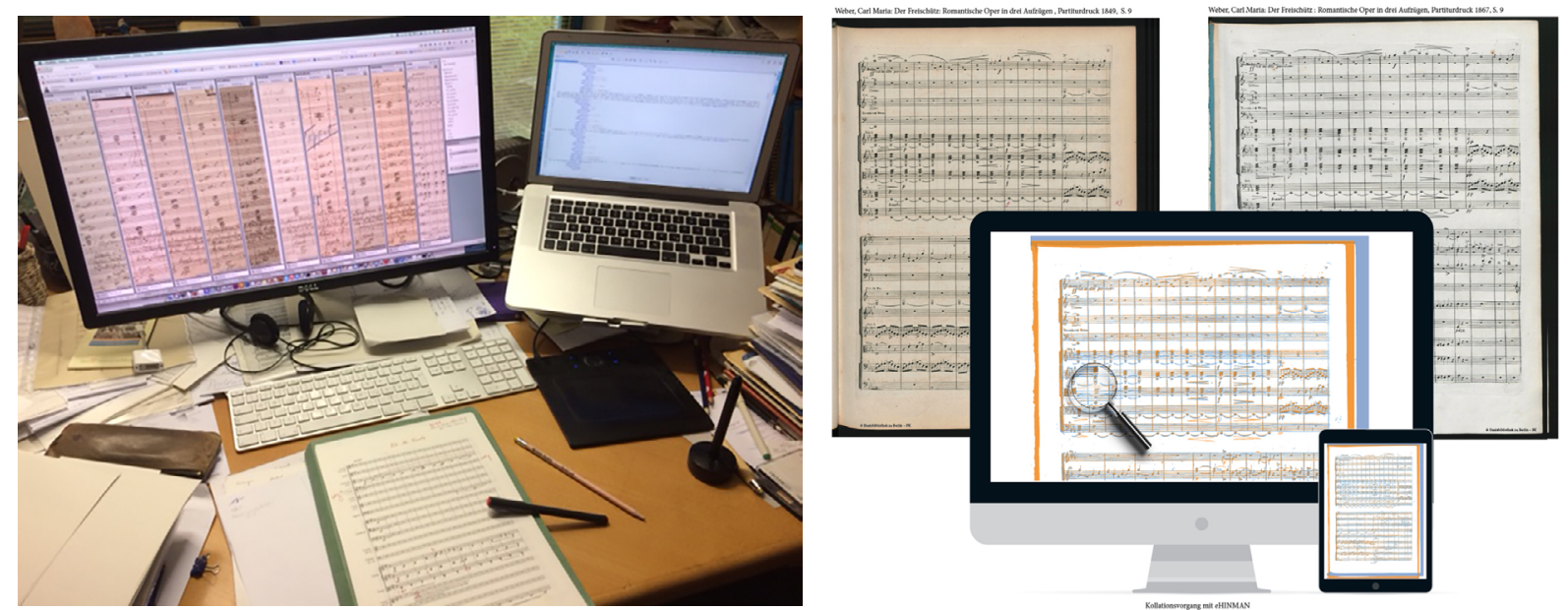

Abbildung 5: Verschiedene Arbeitsumgebungen für Editoren (Quelle: J. Veit/A. Wawilow)

In der linksstehenden Arbeitsumgebung sind gegenüber dem Hinman Collator zusätzliche Funktionen zum räumlichen Arrangieren erkennbar. Auffällig ist dabei, dass dafür hybride Objekte genutzt werden, indem einzelne Takte (ein über die Taktnummer benanntes und digital ansprechbares Objekt) mit einem vertikalen Ausschnitt der jeweiligen Seite einer Notenschrift (digitalisierte Einschreibung) verknüpft werden. Die Taktstreifen sind nicht als digitale Objekte bearbeitbar, sondern können nur gemäß den Anforderungen des Editors arrangiert werden. Im linken Monitor sind einzelne Streifen verschiedener Notenschriften so ausgerichtet, dass sie jeweils denselben Takt anzeigen, um den Vergleich verschiedener Quellen zu unterstützen. Annotationen müssen separat auf Papier oder mit einer anderen Software bearbeitet werden (siehe den separat platzierten Laptop).

Für die Qualität und das Ausmaß der technischen Unterstützungsleistung ist der Übergang von digitalisierten zu digitalen Objekten entscheidend. Dazu müssen Objekte nicht mehr über das Trägermaterial definiert werden, sondern über das Ausschöpfen der technischen Potenziale (vgl. den rechten Bildschirm). Bei digitalisierten Objekten sind die Unterstützungsfunktionen auf Operationen beschränkt, die sich - wie bei analogen Einschreibtechniken - nicht auf den Inhalt beziehen, sondern die Teilung des jeweiligen Trägerformats. So lässt sich z. B. ein Objekt, das in einem digitalen Bildformat vorliegt, zwar in Teilbilder des gleichen Formats zerschneiden und auch visuell anordnen, doch sind die dafür erforderlichen Zusatzdaten nur innerhalb der spezifischen Anwendung vorhanden, denn viele gebräuchliche und standardisierte Grafikfor-

diese aufgrund der Auswertungslogik nicht ohne Weiteres in die traditionellen Schnittstellentechniken integrieren. 
mate bieten keine Möglichkeit, zusätzliche Attribute oder Werte zu integrieren. Insofern kann auch ein spezielles Arrangement nicht als Objekt übertragen werden. Die Konsequenz: das Objekt der Manipulation ist ein optisches Konstrukt nicht eine semantische Einheit, die im Rahmen der Editionsarbeit ein bedeutsames Objekt der Bearbeitung verkörpert.

Dieser Pseudo-Objektbezug hat Konsequenzen für die Arbeit und auch Erweiterung solcher Umgebungen:

- Die durch Schreibhandlungen der Editoren erzeugten Daten sind nur in der jeweiligen Ausprägung der digitalen Arbeitsumgebung persistent, was die Forderung nach Interoperabilität verletzt.

- Semantische Objekte können nicht bearbeitet werden, da sie nicht als digitale Objekte vorliegen. Es gibt zwar eine sichtbare Auszeichnung, mit der man semantisch Eigenschaften verbinden kann, doch können diese Eigenschaften technisch nicht zugeordnet oder ausgewertet werden, da das Arrangement der markierten Objekte seinerseits kein Objekt darstellt, das mit Eigenschaften versehen oder annotiert werden könnte. Auszeichnungen von Objekten wie sie beispielsweise im rechten Bild von Abbildung 5 durch farbliche Werte markiert werden, können nicht objektbezogen erfasst oder ausgewertet werden. ${ }^{30}$

- Durch Medienbrüche, die u. a. entstehen, wenn Objekte in unterschiedlichen Medienformaten vorliegen, entsteht ein enormer kognitiver und motorischer Zusatzaufwand zum Transkribieren und Zusammenführen der jeweiligen Daten, um sie persistent speichern und zu anderen Zeitpunkten weiter bearbeiten zu können.

Einschränkungen dieser Art stehen dem Ausbau von Infrastrukturen für die digitalen Geisteswissenschaften entgegen, lassen sich aber nicht konfliktfrei beseitigen. Um z. B. die Interoperabilität zu gewährleisten, wurden in den letzten Jahrzehnten Standards zum Austausch solcher Daten in digitalen Infrastrukturen erarbeitet. In der Musik ist hier im Kontext historisch-kritischer Editionen an erster Stelle die MEI (Music Encoding Initiative) zu nennen, von der ein Austauschformat zur geräte- und softwareunabhängigen Bearbeitung von Musik entwickelt worden ist. ${ }^{31}$

Sobald digitale Objekte unterschiedliche Repräsentationen haben, kann auch dies zu vielfältigen Medienbrüchen führen. In Abbildung 6 verkörpert die Abbildung links im Vordergrund einen Ausschnitt aus der Syntax von MEI für die Kodierung von Musik. Der Text rechts im Bild entspricht einem minimalen MEI-Dokument, das nur eine Note enthält. Im Hintergrund findet

30 Vgl. hierzu die Beschreibung des gegenwärtig von Anastasia Wawilow zur Feststellung von Differenzen zwischen verschiedenen Abzügen von Druckplatten entwickelten eHinman: <https://ehinman.edirom.de/publishing/Kollationsvorgang_mit_eHinman> (07.08.2020).

31 Bei diesen Austauschformaten - ein weiteres Format ist Music XML - werden alle Daten als lesbarer Text kodiert. MEI wurde speziell für die Anforderungen im wissenschaftlichen Bereich entwickelt; vgl. Kristina Richts und Joachim Veit, "Stand und Perspektiven der Nutzung von MEI in der Musikwissenschaft und in Bibliotheken", in: Bibliothek Forschung und Praxis 42/2 (2018), S. 292-301. 


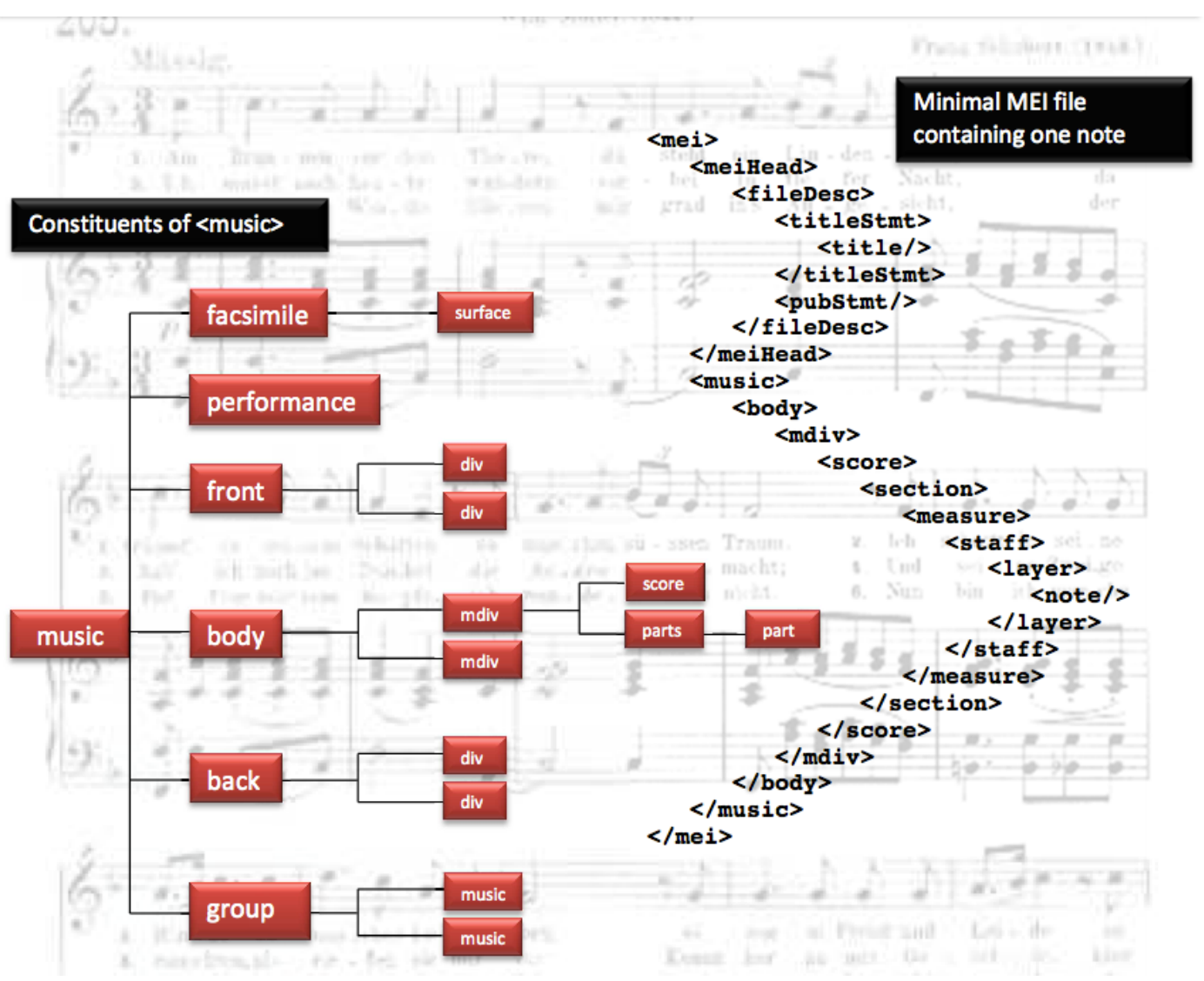

Abbildung 6: Kodierungen von Musik im MEI

sich ein Urtext einer Notenschrift. Alle drei Repräsentationen weisen hinsichtlich ihrer inneren Strukturierung unterschiedliche Objekte auf, die aber auf vielfältige und teilweise subtile Art miteinander verknüpft sind.

Mit der Bearbeitung solch unterschiedlicher Repräsentationen von Musik gehen hohe kognitive und mediale Aufwände einher. Ein und dasselbe semantische Objekt wird bezüglich seiner Aufteilung, Anordnung, Verteilung und semantischer Zuordnung räumlich-visuell unterschiedlich repräsentiert. Neben kryptischen syntaktischen Strukturen und einer starren hierarchischen Anordnung sind Objekte und ihre Beziehungen untereinander teilweise schwer identifizierbar. Zwar gibt es definierte Bausteine wie die einzelnen Notenzeichen oder auch Vorzeichen, doch schon die Frage, was genau ein Notenzeichen an einer bestimmten Stelle im Takt als Objekt ausmacht und wie es bearbeitet werden kann, ist nicht allein lokal bestimmbar. Vorzeichen zu Beginn einer Notenschrift müssen dabei ebenso betrachtet werden wie andere Takte, in denen z. B. ein Vorzeichen aufgehoben oder zusätzlich gesetzt wird.

Sowohl in der Notenschrift wie auch in der MEI-kodierten Fassung ist ein einzelnes Objekt über verschiedene Repräsentationen verteilt, d. h. um ein solches Objekt zu interpretieren, ist es notwendig, Zeichen, die an verschiedenen Positionen stehen, als Einheit zu betrachten. Jedes Schaffen einer Objekteinheit, wenn sie interoperabel sein soll, erfordert jedoch eine 
entsprechende Standardisierung und auch einen Zusatzaufwand in der Kodierung, um solche Objekte spezifizieren und ansprechen zu können. Damit müssen zugleich Fragen nach der Objektgranularität und den vorgesehenen Objektattributen beantwortet werden. Beispielsweise könnten Vorzeichen mit einem Attribut Reichweite versehen werden, um auswerten zu können, welchen nachfolgenden Noten im Tonwert angepasst werden müssen. Es könnten auch Notenzeichen, die über eine Tempoangabe oder ein Zeichen zur Dynamik miteinander verbunden sind, zu Gruppen zusammengefasst werden. Damit zusammen hängt auch das Problem, dass Objekteinheiten auch Anker- und Referenzpunkte für Annotationen oder anderweitige Verknüpfungen von Objekten sein können.

Insgesamt gibt es unzählige Möglichkeiten, die Objektgranularität ebenso wie die Eigenschaften von Objekten zu definieren. Dabei sind Entscheidungen auf unterschiedlichen Ebenen zu treffen, die vom Aufwand zur Kennzeichnung und Erfassung der Attribute von Objekten und der Sicherung von Anschlusshandlungen über die Standardisierung in MEI bis hin zur Implementierung einzelner Werkzeuge und ihre Integration in Forschungsdateninfrastrukturen reichen. ${ }^{32}$

All diese diffizilen Entscheidungen mit ihren Wechselwirkungen ${ }^{33}$ führen dazu, dass jede Definition eines Objekts mit den zugehörigen Funktionen zur Bearbeitung und Auswertung vielfältige Implikationen für Anschlusshandlungen und die Integration in umfassende Infrastrukturen mit sich bringt. Dies gilt umso mehr, wenn man die Objekthaftigkeit im Kontext des ko-aktiven Schreibens betrachtet.

Eine frühe Form des ko-aktiven Schreibens ist Hypertext. Ted Nelson prägte diesen Begriff und charakterisierte inn als nicht-sequentielles Schreiben. ${ }^{34}$ Auf der Basis der Hypertext-Technologie stellte Frans Wiering ein allgemeines multidimensionales Modell für historisch-kriti-

32 Das Problem der Abgrenzung von Objekten und ihrer Granularität ist ein grundsätzliches Problem in den digitalen Geisteswissenschaften, das vom Problem der Objektdefinition (Michael K. Buckland, "What is a ,document'?", in: Journal of the American Society for Information Science 48/9 (1997), S. 804-809 und ders., "What is a ,digital document'?", in: Document Numérique 2/2 (1998), S. 221-230) über die Rolle des analogen Zeichenträgermaterials (Markus Hilgert, Altorientalistik im 21. Jahrhundert - Selbstverständnis, Herausforderungen, Ziele. eine Einführung (= Mitteilungen der deutschen Orient-Gesellschaft zu Berlin 142), Berlin 2010, S. 5-12 <http://www. orient-gesellschaft.de/repositorium/MDOG/MDOG_142.pdf> (12.06.2020), die Kopplung zu hybriden Objekten (Andreas Münzmay, "Lesen und Schreiben im digitalen Dickicht. Musikwissenschaft, Digital Humanities und die hybride Musikbibliothek", in: Bibliothek Forschung und Praxis, 42/2 (2018), S. 236-246) und den Umgang mit unterschiedlichen Medientypen (Ton, Bild, Text) bis hin zu ergonomischen Fragestellungen (Manfred Burghardt, „Annotationsergonomie: Design-Empfehlungen für linguistische Annotationswerkzeuge”, in: Information. Wissenschaft \& Praxis 63/5 (2012), S. 300-304) reicht.

33 Siehe hierzu beispielhaft Joachim Veit, „Die Codierung digitaler Briefeditionen mit TEI P5. Konzepte und Probleme am Beispiel der Carl-Maria-von-Weber-Briefausgabe", in: Digitale Edition zwischen Experiment und Standardisierung, hrsg. von Peter Stadler und Joachim Veit (Beihefte zu editio 31), Tübingen 2009 und ders., „Es bleibt nichts, wie es war" (wie Fn. 2), S. 37-52.

34 Nelsons Leitbild für Hypertext war eine weltweite offene verteilte digitale Bibliothek (bekannt unter dem Namen Xanadu; vgl. Theodor Holm Nelson, "The Unfinished Revolution and Xanadu”, in: ACM Computing Surveys 31/4 (1999), Artikel Nr. 37, <https://doi.org/10.1145/345966.346039>). Allerdings wurde bei vielen Hypertextsystemen dieses Potenzial für die Nutzung nicht ausgeschöpft, weil die technische Realisierung eine Autoren- und eine Lesekomponente bereitstellte. Leser erhielten somit nur eine geringe technische Unterstützung für das nicht-sequentielle Lesen, was auch ohne Technik möglich ist. 
sche Musikeditionen vor. ${ }^{35}$ Die offene Hypertext-Architektur verkörperte die damalige Vorstellung von einem sich kontinuierlich verändernden Korpus von digitalisierten Quellen, digitalen Kodierungen für die algorithmische Bearbeitung sowie Annotationen und Verweisen auf verwandte Arbeiten. Über Lesepfade, die Quellen und Annotationen verknüpfen, sollte es möglich sein, verschiedene Editionen als Sichten auf dieses Korpus zu extrahieren.

Wiering bezieht sich auf die Systemphilosophie des HyperEditing, bei der durch das verteilte Schreiben an einem Hypertext-Korpus ein sich ständig veränderndes Gewebe entsteht, wodurch die Bedeutung des einzelnen Autors sinkt bzw. letztlich ganz verschwindet. ${ }^{36}$ Soweit dies als grundlegende Nutzungsphilosophie gewollt ist, kann man in dieser Form ein offenes Hypertextsystem einsetzen, doch lassen sich differenziertere Nutzungsformen mit unterschiedlichen Rollen und Rechten aufgrund der vielen Wechselwirkungen nicht oder nur mit einem erheblichen Aufwand realisieren. ${ }^{37}$

Annotationen sind ein Schlüsselkonzept für die Arbeit an historisch-kritischen Musikeditionen, für die sich das Konzept Hypertext anbietet, um verteilte Schreibprozesse zu ermöglichen..$^{38}$ Darauf basiert auch das vom W3C standardisierte "open annotation data model" ${ }^{39}$ mit dem ein wachsendes Korpus von Objekten mit Verweisen und Annotationen ko-aktiv erstellt und bearbeitet werden kann. ${ }^{40}$ Offene Annotationen bestehen im Kern aus einem Inhalt (body) und einem Ziel (target). Zwar ist es mit diesem Standard möglich, separat von den Zielobjekten nahezu beliebige Informationen an diese zu knüpfen, doch erhält man mit einer solchen offenen Sammlung miteinander verknüpfter Objekte keine kohärente und vollständige Edition, die als schöpferische Leistung eines Editors unmittelbar sichtbar werden kann. Dieses Problem mag für viele Einsatzbereiche unerheblich sein, jedoch belegen Befragungen von Editoren im

35 Frans Wiering, "Digital Critical Editions of Music: A Multidimensional Model”, zunächst 2006 als Arbeitspapier veröffentlicht: <http://www.methodsnetwork.ac.uk/redist/pdf/wiering.pdf> (12.06.2020). Publiziert mit Überarbeitungen in: Modern Methods for Musicology. Prospects, Proposals, and Realities, hrsg. von Tim Crawford und Lorna Gibson, Ashgate 2009, S. 23-45.

36 Jerome McGann, "Endnote: what is text?”, in: Ma(r)king the Text: The Presentation of Meaning on the Literary Page, hrsg. von Joe Bray, Miriam Handley und Anne C. Henry, London 2000, S. 229-333 und ders., "The Rationale of Hypertext", in: Text 9 (1996), S. 11-32 <http://www.jstor.org/stable/20698008> (10.06.2017).

37 Siehe dazu die kritischen Anmerkungen in Johannes Kepper, Musikedition im Zeichen neuer Medien - Historische Entwicklung und gegenwärtige Perspektiven musikalischer Gesamtausgaben (= Schriften des Instituts für Dokumentologie und Editorik 5), Norderstedt 2011, S. 134ff. Ein anschauliches Beispiel für die Komplexität und die vielfältigen Wechselwirkungen einer hypertextbasierten Konzeption einer digitalen Bibliothek mit der grundlegenden Systemarchitektur von der Datenverwaltung bis hin zur Bereitstellung unterschiedlicher Arten von Verweisen in Kombination mit differenzierten Berechtigungen liefert das Projekt Xanadu (siehe Nelson, „Literary Machines", wie Fn. 24 und "Unfinished Revolution", wie Fn. 34) sowie die journalistische Aufbereitung durch Gary Wolf, "The Curse of Xanadu”, in: Wired 3/6 (Juni 1995), S. 75-85 und 112-113. Die Xanadu zugrundeliegende Vision konnte bis heute nicht erschöpfend umgesetzt werden.

38 Harald Lordick et al., "Digitale Annotationen in der geisteswissenschaftlichen Praxis", in: Bibliothek Forschung und Praxis 40/2 (2016), S. 186-199; zur Komplexität von Annotationen im Bereich der Musik siehe Hadjakos et al., "Challenges for Annotation Concepts in Music", in: International Journal of Humanities and Arts Computing 11/2 (2017), S. 255-275.

39 <http://www.openannotation.org/spec/core/20130208/index.html> (12.06.2020).

40 Lordick et al., "Digitale Annotationen”. 
Bereich historisch-kritischer Musikeditionen, dass sie mit einer solchen, gewissermaßen durch die Hintertür eingeführten Anonymisierung nicht einverstanden wären. ${ }^{41}$

In der Konsequenz bedeutet dies, dass sich der Objektcharakter auf verschiedenen Ebenen unterschiedlich manifestiert und sich die damit verbundenen Anforderungen nicht konfliktfrei umsetzen lassen. Verschärft wird diese Situation noch durch die Tatsache, dass Objekte nicht nur von verschiedenen Personen hinzugefügt, sondern auch bearbeitet werden können. In welcher Kombination genau welche Berechtigungen für welche Ausschnitte einer verteilten multimedialen Edition vergeben werden sollen, lässt sich entsprechend ebenfalls nicht konfliktfrei lösen, denn die beteiligten Akteure und Institutionen haben nicht nur unterschiedliche Ziele, sondern damit zusammenhängend auch unterschiedliche Aufgaben.

Speziell mit Blick auf das Potenzial des ko-aktiven Schreibens wird offenbar, dass eine virtuelle Forschungsumgebung kein lokal installierbares Einzelwerkzeug verkörpert, sondern immer Teil einer komplexen Infrastruktur mit vielfachen Wechselwirkungen zwischen Technik und Nutzungsformen ist. Aggregierte Erhebungen von Wünschen und Anforderungen ${ }^{42}$ schaffen $^{2}$ allein noch keine ausreichende Grundlage zur Verständnisbildung in Projekten der digitalen Geisteswissenschaften, da sie weder wichtige Wechselwirkungen noch entscheidende Gestaltungskonflikte thematisieren.

Dies soll kurz an der Gestaltungsdimension "Artikulieren" skizziert werden.

Mit Artikulation ist der Schreibprozess an einem primären Objekt gemeint. Das sind solche Objekte, die ko-aktiv erstellt und bearbeitet werden können, wie z. B. ein gemeinsam von mehreren Autoren verfasster Aufsatz. Das Objekt Aufsatz verkörpert einen eigenen Handlungsraum, in dem alle Beteiligten die gleichen Berechtigungen insbesondere zum Schreiben haben. Da grundsätzlich alle Gestaltungsdimensionen unterschiedliche Stufen der Ausprägung zulassen, lässt sich auch ein solcher Handlungsraum unterschiedlich ausgestalten. Auf der untersten Stufe gibt es keine Differenzierung, $d$. h. alle Funktionen und Berechtigungen sind für alle Beteiligten gleichermaßen zugreifbar. Auf der nächsten Stufe könnte man den Handlungsraum segmentieren, sodass man ein Segment mit unterschiedlichen Attributen belegen kann. In Kombination mit anderen Gestaltungsdimensionen wie z. B. Berechtigen kann man nun differenzierte Schreib- und Leserechte für ein Segment vorsehen, sodass auf diese Weise die Nutzungsform eines Blogs umsetzbar wäre, ${ }^{43}$ bei dem Autoren Artikel auf einer Webseite publizieren, die von allen Lesern kommentiert werden können. Diese Kommentare werden meist am Ende chrono-

41 Bianca Meise und Dorothee M. Meister, „Digitale Transformationen. Zum Einfluss der Digitalisierung auf die musikwissenschaftliche Editionsarbeit", in: merz. medien + erziehung 66/6 (2017), S. 42-51.

42 Thomas Süptitz, Stephan J. J. Weis und Torsten Eymann, „Was müssen Virtual Research Environments leisten? Ein Literaturreview zu den funktionalen und nichtfunktionalen Anforderungen", in: 11th International Conference on Wirtschaftsinformatik, Leipzig 2013, S. 327-341.

43 Dabei sei daran erinnert, dass sich die Entscheidung, welche Segmente es geben soll und mit welchen Berechtigungen sie aktuell ausgestattet werden, zur Nutzungszeit passiert. 
logisch aufgeführt. Über die Berechtigungen wird ein gemeinsamer Wahrnehmungsraum mit einem segmentierten Handlungsraum konstruiert.

Eine solche Aufteilung allein wäre jedoch für die Arbeit mit ko-aktiven Musikeditionen bei weitem nicht ausreichend, denn schließlich ändern sich Objekte im Prozess der ko-aktiven Wissensarbeit ebenso wie die Personen, die sich mit innen befassen. Diskrepanzen tauchen auf, Einsichten müssen revidiert und Daten müssen angepasst, gelöscht oder ergänzt werden. Die mangelnde Persistenz digitaler Objekte schafft Vorteile für die flexible Bearbeitung, doch muss man auch vorsehen, dass Varianten eines Objektes oder Versionen entstehen, die selbst als Objekte dem weiteren Forschungsprozess zugänglich sein sollten. Dabei wird jetzt nicht der Handlungsraum segmentiert, sondern die Objekte im Handlungsfeld der Forschenden treten in unterschiedlichen, gleichwohl aufeinander bezogenen Ausprägungen auf, die beispielsweise wieder mit unterschiedlichen Berechtigungen verbunden sein können oder mit zusätzlichen Attributen, die die Urheberschaft kennzeichnen. Die technische Verwaltung dieser Daten ebenso wie die entsprechenden Auswertungsfunktionen werfen erneut Wechselwirkungen und Gestaltungskonflikte auf, die teilweise mit herkömmlichen Mitteln nicht oder nur sehr unzureichend bewältigt werden können.

Ein kurzes Beispiel soll diese Problematik verdeutlichen. Im analogen Medienbereich wird der Handlungsraum über physische Objekte und Geräte zur Vorführung oder Bearbeitung des Trägermaterials charakterisiert. Inschriften sind per definitionem persistent und viele der Nutzungsformen basieren darauf, dass es sich in der Regel jeweils um lokale Objekte bzw. Kopien von Objekten handelt. Wie aber soll man mit dauerhaft digital vorhandenen Objekten verfahren, um sowohl die nachhaltige Persistenz als auch zugleich die Bearbeitbarkeit bzw. auch das Ausgliedern von Objekten zu ermöglichen?

Mit offenen Verweisstrukturen, wie sie bislang beschrieben worden sind, ist es nicht möglich, geeignete Infrastrukturen aufzubauen. So lassen sich beispielsweise Aspekte der Vertrauenswürdigkeit verteilter Objekte damit technisch nicht abbilden. Ein Verweis mag ein definiertes Objekt eindeutig referenzieren, doch ob dieses Objekt tatsächlich das vermutete und zugleich unverfälscht ist, lässt sich mit dieser Technik nicht sichern, sondern hängt von der Vertrauenswürdigkeit der speichernden bzw. das Objekt verwaltenden Einrichtung ab. Ebenso lassen sich zeitliche Zusammenhänge, z. B. welches Objekt zuerst vom wem geschrieben worden ist (Urheberschaft), damit nicht abbilden bzw. modellieren. Dies ist aber von essentieller Bedeutung, will man Editionsinfrastrukturen ermöglichen, bei denen ein (semantisches) Objekt als Handlungseinheit verteilt und ko-aktiv bearbeitet werden kann.

Um solche weitreichenden Herausforderungen angehen zu können, muss man deshalb neue technische Lösungen entwickeln und dabei auch bereits vorhandene Techniken wie z. B. Versionsverwaltungssysteme oder Blockchains hinsichtlich ihrer Potenziale und Wechselwirkungen für Editionsinfrastrukturen bewerten. 
Mit Hilfe kryptografischer Verfahren, bei denen ein Wert aus dem Objektinhalt berechnet wird, und mit einem digitalen Zeitstempel, mit dem die Existenz und Echtheit eines Objekts zu einem bestimmten Zeitpunkt nachgewiesen werden kann, ${ }^{44}$ lassen sich zwei Attribute für jedes Objekt angeben, um die Authentizität und Urheberschaft digitaler verteilter Objekte zu sichern.

Unter Einbeziehung des Konzepts der Revisionssicherheit hat Andreas Oberhoff 2020 eine stringente Aufbereitung mit sehr differenzierten Überlegungen zu dieser Problematik vorgelegt. Dabei macht er deutlich, dass auch mit der Identifizierung technischer Potenziale noch keine Gestaltungslösung verbunden ist, bzw. diese deduktiv aus Anforderungen abgleitet werden kann, denn auch diese technischen Lösungsansätze bergen Gestaltungskonflikte, die technikimmanent nicht gelöst werden können, sie erfüllen nur einen Teil der Anforderungen und haben zugleich auch Nachteile. Nutzt man beispielsweise die gerade angesprochenen Verfahren im Rahmen eines Blockchain-Ansatzes, ${ }^{45}$ muss man sich darauf einstellen, dass es nicht ohne Weiteres möglich ist, ein Objekt in der Blockchain zu löschen.

\section{Resümee}

Digitale vernetzte Systeme mit einer programmierten Schnittstelle ermöglichen es in einem nie zuvor gekannten Ausmaß, Handlungs- und Wahrnehmungsraum bei der Wissensarbeit möglichst eng aber zugleich auch flexibel miteinander zu koppeln. Entscheidungen darüber, was als Objekt identifiziert und mit entsprechenden Funktionen bearbeitet werden kann, wurden im Verlaufe der Computerentwicklung zunehmend vom Zeitpunkt der Entwicklung eines technischen Systems in den Zeitraum seiner Nutzung verlegt. Diese grundsätzliche Tendenz, die sich bis ins Internet der Dinge fortsetzt, reduziert das Antizipationsdefizit bei der Gestaltung, vergrößert die Flexibilität in der Anwendung und eröffnet zugleich neue Möglichkeiten in der Nutzung.

Um im Kontext von Projekten in den digitalen Geisteswissenschaften generell und der $\mathrm{Mu}$ sikwissenschaft im Besonderen aus technischen Potenzialen alltagstaugliche Mehrwerte zu schöpfen, reicht es nicht aus, nach generischen Werkzeugen und Diensten Ausschau zu halten, die unabhängig vom aktuellen Kontext der Wissensarbeit nutzbar sind. Schon in Bezug auf die Spezifika in einzelnen geisteswissenschaftlichen Disziplinen gibt es entscheidende Unterschiede, die es zu berücksichtigen gilt. ${ }^{46}$

Hinzu kommt, dass für die Entwicklung von Forschungsinfrastrukturen eine Fülle von Wechselwirkungen zu berücksichtigen und unzählige Gestaltungskonflikte auszutarieren sind.

44 Stuart Haber, W. Scott Stornetta, "How to Time-Stamp a Digital Document”, in: Advances in Cryptology - CRYPTO '90, hrsg. von Alfred J. Menezes und Scott A. Vanstone, Berlin und Heidelberg 1991, S. 437-455.

45 Andreas Oberhoff, Revisionssicherheit von Forschungsdateninfrastrukturen (wie Fn. 2), S. $237 \mathrm{ff}$.

46 Joachim Veit stellt beispielsweise die Frage: „Musikwissenschaft und Computerphilologie - eine schwierige Liaison?", in: Jahrbuch für Computerphilologie 7 (2006), S. 67-92, und erhofft sich angesichts der zukünftigen Herausforderungen trotz aller Differenzen eine kluge Liaison. 
Das bedeutet, dass berechtigte Anforderungen nur auf Kosten anderer gleichermaßen berechtigter Anforderungen umgesetzt werden können. Die Entscheidungen jedoch, welche Anforderungen vollständig, partiell oder zugunsten anderer Anforderungen nicht umgesetzt werden, hängt in vielen Fällen vom Nutzungskontext ab. In manchen Fällen kann es erwünscht sein, offene Nutzergruppen zu haben, in anderen nicht. Die Vergabe von Berechtigungen kann flexibel oder restriktiv gehandhabt werden, und je nach technischer Umsetzung erhöht sich der Verwaltungsaufwand für die Rechteerteilung und -verwaltung. Wie kann eine MEI-Datei in Ausschnitten verfügbar gemacht werden, um Rechtsverletzungen auszuschließen, zugleich aber möglichst vielfältige Nutzungsformen zu ermöglichen? Solche Entwurfsentscheidungen sind keine "Entweder-Oder"-Entscheidungen, sondern schwierige Abwägungsprozesse in denen graduell berechtigte aber im Widerspruch zueinander stehende Anforderungen austariert werden müssen. Solche Entscheidungen sind mit Werthaltungen verbunden und daher nicht von vornherein inhaltsneutral.

Die hier vorgestellten Ansätze für eine hypothesengeleitete Technikgestaltung haben das Ziel, Wissen, Konzepte und Methoden aus der Informatik anschlussfähig aufzubereiten um im transdisziplinären Diskurs Begründungszusammenhänge für Entwurfsentscheidungen transparent zu machen. Zugleich sollen sie eine konstruktive Unterstützung bieten, um das Antizipationsdefizit bei der Gestaltung zu verringern. Da enorm viele Begriffe der Informatik metaphorische Anleihen aus nicht-technischen Bereichen sind (Information, Kooperation, Intelligenz, Dialogsystem, etc.) und damit die Gefahr der impliziten Unterstellung einer funktionalen Äquivalenz von menschlicher Informationsverarbeitung und maschineller Datenverarbeitung gegeben ist, kommt einer kontrastiven Begriffsbildung ein besonderer Stellenwert zu. Sie kann helfen, Missverständnisse und falsche Erwartungen an technische Systeme aufzudecken. Längerfristig könnten sie auch eine Grundlage sein, um die Anschlussfähigkeit zu Desideraten nicht-technischer Disziplinen, wie z. B. den eingangs angesprochenen Scholarly Primitives herzustellen.

Zitation: Reinhard Keil, „Der Computer als Denkzeug für hermeneutische Arbeit”, in: Brückenschläge zwischen Musikwissenschaft und Informatik. Theoretische und praktische Aspekte der Kooperation, in Verbindung mit der Fachgruppe Digitale Musikwissenschaft hrsg. von Stefanie Acquavella-Rauch, Andreas Münzmay und Joachim Veit (= Musikwissenschaft: Aktuelle Perspektiven. Bericht über die Jahrestagung der Gesellschaft für Musikforschung 2019 in Paderborn und Detmold, Bd. 3), Detmold, Musikwissenschaftliches Seminar der Universität Paderborn und der Hochschule für Musik Detmold, 2020, S. 3-26, DOI: 10.25366/2020.89 


\section{Abstract}

Computer science and the humanities seem to belong to two opposite sides within the spectrum of scientific methods and research. In the domain of digital humanities, however, formalization and hermeneutic interpretation have to be integrated. As will be argued in this article, this integration provides a fundamentally new challenge to both disciplines.

In particular, researchers from the humanities want to be sure that using the tools provided by computer science (big data, machine learning, etc.) do not change insights in any non-expected way. However, even if this could be partially secured, it cannot be achieved in general for most of the research practices. As will be demonstrated in the context of digital editions in musicology, it is impossible to design technology in a neutral or context-free manner. Due to the interests of different actors and institutions, numerous design conflicts arise where the implementation of requirements violates other, equally valid demands. To balance these conflicting requirements invariably brings some bias to the overall design. Thus, it is important to develop a strategy for identifying potential influences as well as the impact of design decisions throughout the whole process of developing tools and infrastructures.

The paper presents an approach for hypotheses driven design of digital tools and infrastructures from a computer science point of view, placing great emphasis on supporting mutual understanding and ensuring a transparent design process.

\section{Kurzvita}

Prof. Dr. Reinhard Keil, Professor für Kontextuelle Informatik am Heinz Nixdorf Institut der Universität Paderborn (1992-2019). Forschungsthemen: Computer und Gesellschaft, E-Learning, Gestaltung digitaler Medien und verteilte Wissensorganisation. Promotion (1984) und Habilitation (1991) in der Informatik an der TU Berlin. Von 1990-1991 Forschungsaufenthalt an der University of Maryland, College Park. Von 2015-2019 Dekan der Fakultät für Elektrotechnik, Informatik und Mathematik der Universität Paderborn. 



\section{Brückenschläge zwischen Musikwissenschaft und Informatik}

Theoretische und praktische Aspekte der Kooperation

Herausgegeben von Stefanie Acquavella-Rauch,

Andreas Münzmay und Joachim Veit 
Brückenschläge zwischen Musikwissenschaft und Informatik 


\section{Musikwissenschaft: Aktuelle Perspektiven}

Bericht über die Jahrestagung der Gesellschaft für Musikforschung 2019 in Paderborn und Detmold

Herausgegeben von Rebecca Grotjahn und Nina Jaeschke

Band 3 


\section{Brückenschläge zwischen Musikwissenschaft und Informatik}

Theoretische und praktische Aspekte der Kooperation

Beiträge der Symposien zur Digitalen Musikwissenschaft

Osnabrück 2018 und Paderborn 2019

im Rahmen der Jahrestagungen der Gesellschaft für Musikforschung

In Verbindung mit der Fachgruppe Digitale Musikwissenschaft herausgegeben von

Stefanie Acquavella-Rauch, Andreas Münzmay und Joachim Veit

Detmold: Musikwissenschaftliches Seminar der Universität Paderborn und der Hochschule für Musik Detmold 2020 
DOI: $10.25366 / 2020.87$

Online-Version verfügbar unter der Lizenz: Urheberrecht 1.0, $<$ https://rightsstatements.org/page/InC/1.0/?language =de >

Bibliografische Information der Deutschen Nationalbibliothek

Die Deutsche Nationalbibliothek verzeichnet diese Publikation in der Deutschen Nationalbibliografie; detaillierte bibliografische Daten sind im Internet über http://dnb.d-nb.de abrufbar.

\section{Impressum}

Redaktion: Stefanie Acquavella-Rauch, Andreas Münzmay und Joachim Veit Satz: Nina Jaeschke und Joachim Veit

(C) Musikwissenschaftliches Seminar der Universität Paderborn und der Hochschule für Musik Detmold 2020 


\section{INHALT}

Rebecca Grotjahn, Nina Jaeschke

Vorwort zu Band 1-3

IX

Stefanie Acquavella-Rauch, Andreas Münzmay, Joachim Veit

Brückenschläge zwischen Musikwissenschaft und Informatik - Vorbemerkung

$\mathrm{XI}$

\section{KOLLABORATIONEN - KO-LABORATORIEN}

\section{Reinhard Keil}

Der Computer als Denkzeug für hermeneutische Arbeit

\section{Ulrich Konrad}

Philologie und Digitalität. Perspektiven für die Musikwissenschaft im Kontext fächerübergreifender Institutionen

\section{Gudrun Oevel}

Infrastruktureinrichtungen in Forschungsprojekten - Spagat oder Chance?

\section{Dennis Ried}

Erhebung, Transformation und Präsentation digitaler Forschungsdaten

Anna Neovesky, Frederic von Vlahovits

IncipitSearch - Leitfaden zur Zusammenarbeit

Elisabeth Treydte

Clara Schumann \#digital. 40 Jahre Archiv Frau und Musik und der Start in die Digitalisierung

\section{TEXT/DATEN/PROZESSE}

\section{Christine Siegert}

Komponisten-Gesamtausgaben im digitalen Zeitalter: Perspektiven

und Reflexionen am Beispiel Ludwig van Beethovens

Markus Neuwirth, Johannes Hentschel, Martin Rohrmeier

Perspectives of Musical Corpus Studies: The Annotated Mozart Sonatas

Agnes Amminger, Franz Kelnreiter

Leopold Mozarts „Gründliche Violinschule". Zur Textcodierung und -präsentation einer digitalen Edition

\section{Oleksii Sapov}

Algorithmische Automatisierung komplexer Notationsregeln in MEI-XML am Beispiel von Versetzungszeichen 


\section{Susanne Cox, Richard Sänger}

Digitale Fassungsvergleiche am Beispiel von Beethovens Eigenbearbeitungen

Agnes Seipelt

Digitale Edition und Harmonische Analyse mit MEI von Anton Bruckners

Studienbuch

Stefanie Acquavella-Rauch

Musikalische Schaffensprozesse 2.0 - Inkorporation audiovisueller Medien

der populären Musik in Methoden der digitalen Edition

\section{DIGITAL(ISIERT)E MATERIALITÄTEN}

\section{Miriam Akkermann}

(Musik)Instrument (im) Computer

\section{Daniel Fütterer}

Herausforderungen bei der Kodierung von Paratext am Beispiel Neuer Musik mit Live-Elektronik

\section{Matthias Pasdzierny}

How much is the glitch? Das digitale Paradigma als Herausforderung

und Chance für die historische Musikwissenschaft

\section{Shintaro Miyazaki}

Musik für Maschinen?! - Wo sich die Wissenschaft der Medien, des Computers und der Musik treffen und wie sie zusammenarbeiten könnten

\section{MUSIKGESCHICHTE(N) IM NETZ}

\section{Matthias Tischer}

Musikgeschichte der DDR: Ein Pilotprojekt zur digitalen Musikvermittlung

Annette van Dyck-Hemming, Jan Eberhardt, Melanie Wald-Fuhrmann

Ansätze zur Analyse historischer Netzwerke mit Neo4j® - Aus der Projekt-Werkstatt der Datenbank zur Fachgeschichte der Musikwissenschaft

Axel Beer, Martin Bierwisch, Kristina Krämer

Das MMM2 - Ein regionalgeschichtliches Onlinelexikon der Arbeitsgemeinschaft für mittelrheinische Musikgeschichte

\section{Matej Santi}

Was erzählt Fritz Kreislers Geige?

\section{Elias Berner}

Alle Menschen werden Brüder?! Ein historisches Dokument aus dem

Nationalsozialismus in den sozialen Medien 
Gabriele Buschmeier in memoriam 